\title{
GRAVITY DRIVEN SHALLOW WATER MODELS FOR ARBITRARY TOPOGRAPHY *
}

\author{
FRANÇOIS BOUCHUT ${ }^{\dagger}$ AND MICHAEL WESTDICKENBERG $\ddagger$
}

\begin{abstract}
We derive new models for gravity driven shallow water flows in several space dimensions over a general topography. A first model is valid for small slope variation, i.e. small curvature, and a second model is valid for arbitrary topography. In both cases no particular assumption is made on the velocity profile in the material layer. The models are written for an arbitrary coordinate system, and several formulations are provided. A Coulomb friction term is derived within the same framework, relevant in particular for debris avalanches. All our models are invariant under rotation, admit a conservative energy equation, and preserve the steady state of a lake at rest.
\end{abstract}

Key words. Shallow water - arbitrary topography - lake at rest - energy equation - velocity profile - debris avalanche.

\section{Introduction}

The problem of modeling gravity driven shallow water flows arises in many physical situations, such as weather forecast, ocean modeling, flows in rivers and coastal areas, debris avalanches, etc. Consider the motion of a relatively thin layer of material under the influence of gravity over a complex relief, like the ground of an ocean, or over a mountain. Our particular interest here is to take into account as much as possible the influence of the topography in the flow equations. While the dynamics are well understood for the flat case, and also the mathematical theory becomes settled now, see $[8,7,6,1]$, the situation is different for the nonflat case. Several models are discussed in the literature, that we briefly describe now.

The classical Saint-Venant system [12] is given by

$$
\begin{gathered}
\partial_{t} h+\partial_{x}(h u)=0, \\
\partial_{t}(h u)+\partial_{x}\left(h u^{2}+\frac{1}{2} g h^{2}\right)+h g \partial_{x} z=0 .
\end{gathered}
$$

It is widely used to model flows in one space dimension. Here $h$ is the height of the material, $u$ is the velocity in the direction parallel to the bed, and $g$ is the gravity constant. The influence of the topography enters through the function $z(x)$ which is the altitude of the relief. The Saint-Venant system is derived from the free surface incompressible Navier-Stokes equations in the layer in the regime of small slopes, i.e. with $\partial_{x} z$ being small. It is robust, hyperbolic and admits a convex entropy, the energy of the system. One important feature of it is that the steady state of a lake at rest, i.e. $h+z=$ const and $u=0$, is preserved.

In 1991 Savage and Hutter [13] introduced a model which is capable of handling more general slopes. In its adjusted form, it is given by

$$
\begin{gathered}
\partial_{t} h+\partial_{X}(h u)=0 \\
\partial_{t}(h u)+\partial_{X}\left(h u^{2}+\frac{1}{2} h^{2} g \cos \theta\right)+h g \partial_{X} z+\frac{1}{2} g h^{2} \partial_{X}(\cos \theta)=0 .
\end{gathered}
$$

*Received: May 10, 2004; accepted (in revised version): June 20, 2004. Communicated by Lorenzo Pareschi. This work was realized during the visit of Michael Westdickenberg at ENS/DMA with support by the European network Hyperbolic and kinetic equations, funded by the EC as contract HPRN-CT-2002-00282.

${ }^{\dagger}$ CNRS \& Département de Mathématiques et Applications, École Normale Supérieure, 45 rue d'Ulm, 75230, Paris cedex 05, France (fbouchut@dma.ens.fr).

${ }^{\ddagger}$ Institute for Applied Mathematics, University of Bonn, Wegelerstr.10, 53115 Bonn, Germany (mwest@iam.uni-bonn.de). 
The momentum equation can be replaced, for smooth solutions, by the velocity equation

$$
\partial_{t} u+\partial_{X}\left(\frac{1}{2} u^{2}+h g \cos \theta+g z\right)=0 .
$$

Again the model is one-dimensional. But unlike the Saint-Venant equations which are set up in cartesian coordinate $x$, the Savage-Hutter model uses the curvilinear coordinate $X$ along the topography. The function $\theta(X)$ measures the angle of the bed tangent with the horizontal reference frame, thus

$$
\frac{d x}{d X}=\cos \theta,
$$

and we have the relations

$$
\frac{d z}{d x}=\tan \theta, \quad \frac{d z}{d X}=\sin \theta .
$$

The Savage-Hutter model is valid in the regime of small slope variation, i.e., for $\theta_{X} \equiv \partial_{X} \theta$ small. Note that $\theta_{X}$ is the curvature of the terrain. As before, $h$ is the width of the material layer, this time measured in normal direction, not vertically. Still $u$ is the tangential velocity. The system is hyperbolic, admits a convex entropy (the energy) and preserves the lake at rest steady state $u=0, h \cos \theta+z=$ const. It is widely used, e.g. for the modeling of debris avalanches, in which case a suitable friction term is added.

Recently, Bouchut et al. [2] derived a set of equations which models gravity driven shallow water flows in one space dimension, but without any restriction on the topography,

$$
\begin{gathered}
\partial_{t}\left(h-\frac{1}{2} \theta_{X} h^{2}\right)+\partial_{X}\left(\frac{\ln \left(1-h \theta_{X}\right)}{-\theta_{X}} W\right)=0, \\
\partial_{t} W+\partial_{X}\left(\frac{W^{2}}{2\left(1-h \theta_{X}\right)^{2}}+h g \cos \theta+g z\right)=0 .
\end{gathered}
$$

Again the curvilinear coordinate $X$ is used, $h(t, X)$ is the width in normal direction, and $W(t, X)$ is related to the tangential velocity profile $u$ defined in the material layer by

$$
u(t, X, \bar{\xi})=W(t, X) /\left(1-\bar{\xi} \theta_{X}\right),
$$

where $0 \leq \bar{\xi} \leq h(t, X)$ is the normal variable. The system admits a convex entropy and preserves the lake at rest steady state $W=0, h \cos \theta+z=$ const. The model contains asymptotically the Savage-Hutter model under the assumption $\theta_{X}=\mathcal{O}(\epsilon)$, by neglecting terms in $\epsilon^{3}$ and $\epsilon^{2}$ respectively in (1.6). The number $\epsilon$ is the aspect ratio between the width of the layer and the typical length of phenomena in $x$ (thus by definition $h=\mathcal{O}(\epsilon))$.

In the multidimensional case the models are less developed. This is mainly due to the complexity of the geometry. The curvature, for example, becomes a matrix, so the quantities that need to be included into the model are much more difficult to guess than in one dimension. The extension to multidimension of the Saint-Venant system is of course obvious, but it is valid only for almost flat topography, thus not relevant for debris avalanches in particular. The extension to several dimensions of the 
Savage-Hutter model is nontrivial. The first attempt has been made by Gray, Wieland and Hutter [5]. Their model assumes that the topography has large variation only in one direction, while it is essentially flat in the other direction. Variants of this model can be found in [14], [4]. Very recently, Hutter and Pusadaini introduced a model for avalanches in arbitrarily curved and twisted channels, see [11] and [10]. However, to our knowledge, there still exists no truly multidimensional model for gravity driven shallow water flows on a general topography.

Our first aim in this paper is to provide general equations extending the SavageHutter theory to several space dimensions. With the same approach, we also generalize the model of [2] without the small curvature assumption. A new argument that we also propose enables us indeed to remove the assumption on the velocity profile in the normal variable (constant for (1.2), specific linear dependence (1.7) for (1.6)). It is replaced by the interpretation that the velocity variable is approximately the average value in the layer of the velocity profile.

In view of the fact that the model must be solved numerically, it is desirable to have some flexibility in the choice of (possibly curvilinear) coordinates, thus our models are written in arbitrary coordinates. Our models also inherit the following features from the incompressible Navier-Stokes equations with free surface:

- The systems admit a conservative entropy equation. This ensures hyperbolicity, and this is particularly important in order to describe shock formation, and for numerical stability.

- The models preserve the steady state of a lake at rest.

- The models are invariant by rotation.

- The models imply intrinsically that the vorticity is transported by the flow. However, in contrast with [13], we shall not take into account internal angles of friction.

The paper is organized as follows. In Section 2 we state our models and their intrinsic properties, including the case of Coulomb friction. In Section 3 we derive the models from the free surface incompressible Navier-Stokes equations. Finally, in Section 4 we give a detailed justification of the invariance under rotation.

\section{Multidimensional shallow water models}

This section is devoted to the introduction of our models for multidimensional gravity driven shallow water flows valid for general geometries. Our main model in Subsection 2.2 is valid under the assumption of small slope variation, or equivalently of small curvature, while the model proposed in Subsection 2.3 is more general and is valid without this assumption, generalizing the one-dimensional model of [2]. The Coulomb friction term is described in Subsection 2.4 for both cases.

2.1. Topography description. We assume that the topography is given as the graph of some scalar function $z(x)$, the altitude of the terrain, where $x \in \mathbb{R}^{N}$ is the horizontal coordinate, see Figure 2.1, the physically relevant cases being $N=1$ or $N=2$. We denote the graph by $\mathcal{S} \subset \mathbb{R}^{N+1}$. We assume $z$ sufficiently smooth, so that the unit upward normal vector $\vec{n}$ to $\mathcal{S}$ can be defined. It is given by

$$
\vec{n}=\left(-\frac{\nabla_{x} z}{\sqrt{1+\left|\nabla_{x} z\right|^{2}}}, \frac{1}{\sqrt{1+\left|\nabla_{x} z\right|^{2}}}\right) \equiv(-\mathbf{s}, c) \in \mathbb{R}^{N} \times \mathbb{R} .
$$

The scalar $c>0$ is indeed the cosine of the angle between $\vec{n}$ and the vertical. From these definitions of $\mathbf{s}(x)$ and $c(x)$, and since $|\mathbf{s}|^{2}+c^{2}=1$, we notice that

$$
\partial_{x} c=-\frac{1}{c} \mathbf{s}^{t} \partial_{x} \mathbf{s},
$$




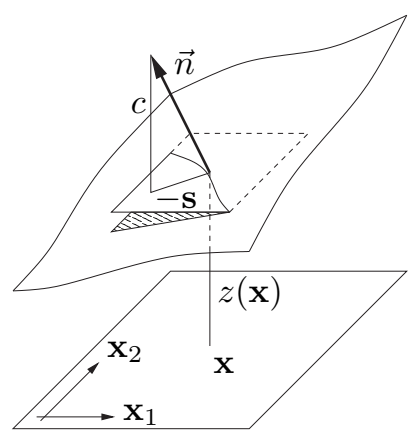

FIG. 2.1. Multidimensional topography

$$
\partial_{x} \mathbf{s}=c\left(\operatorname{Id}-\mathbf{s s}^{t}\right) \partial_{x x}^{2} z, \quad \partial_{x x}^{2} z=\frac{c^{2} \mathrm{Id}+\mathbf{s} \mathbf{s}^{t}}{c^{3}} \partial_{x} \mathbf{s},
$$

where we used the identity $\left(\mathrm{Id}-\mathbf{s} \mathbf{s}^{t}\right)\left(\mathrm{Id}+\mathbf{s s}^{t} / c^{2}\right)=\mathrm{Id}$. The matrix

$$
\mathcal{H}=c^{3} \partial_{x x}^{2} z
$$

is the curvature tensor of $\mathcal{S}$.

It is sometimes convenient not to work in cartesian coordinates, but in a coordinate system which is adapted to the topography. We account for that need by assuming that we are given a parametrization of $\mathcal{S}$, or equivalently of the horizontal coordinate $x \in \mathbb{R}^{N}$ by a curvilinear coordinate $\boldsymbol{\xi} \in \mathbb{R}^{N}$, i.e., we have a bijection

$$
\boldsymbol{\xi} \mapsto \mathbf{x}(\boldsymbol{\xi}) \in \mathbb{R}^{N}, \quad \boldsymbol{\xi} \in \mathbb{R}^{N} .
$$

We denote by $\partial_{\boldsymbol{\xi}} \mathbf{x}$ the Jacobian matrix of $\mathbf{x}$, and for convenience we assume that $\operatorname{det} \partial_{\boldsymbol{\xi}} \mathbf{x}>0$. We shall write down our models in general $\boldsymbol{\xi}$-coordinates, but of course it is possible to make the choice $\mathbf{x}(\boldsymbol{\xi})=\boldsymbol{\xi}$. Anyway, the models are independent of the chosen coordinates.

2.2. A multidimensional shallow water model for small slope variation.

The model reads as follows. The flow is described by

$$
h(t, \boldsymbol{\xi}) \geq 0, \quad V(t, \boldsymbol{\xi}) \in \mathbb{R}^{N},
$$

where $h$ is the width of the material layer in direction normal to the terrain, and $V$ parameterizes the velocity field, in the sense that defining

$$
\mathcal{V}=\left(\partial_{\boldsymbol{\xi}} \mathbf{x}\right) V
$$

the material velocity vector $\mathcal{V}^{t g} \in \mathbb{R}^{N+1}$ has horizontal/vertical components

$$
\mathcal{V}^{t g}=\left(\begin{array}{c}
\mathrm{Id} \\
\frac{1}{c} \mathbf{s}^{t}
\end{array}\right) \mathcal{V}
$$

This formula shows obviously that this physical velocity is tangent to the topography, $\mathcal{V}^{t g} \cdot \vec{n}=0$. Its norm is $\left|\mathcal{V}^{t g}\right|^{2}=|\mathcal{V}|^{2}+\left(\mathbf{s}^{t} \mathcal{V} / c\right)^{2}$.

Our model reads as two equations on $h$ and $V$,

$$
\partial_{t}\left(J_{0} h\right)+\nabla_{\boldsymbol{\xi}} \cdot\left(J_{0} h V\right)=0,
$$




$$
\partial_{t} V+V \cdot \nabla_{\boldsymbol{\xi}} V+\left(\partial_{\boldsymbol{\xi}} \mathbf{x}\right)^{-1}\left(\mathrm{Id}-\mathbf{s s}^{t}\right)\left(\partial_{\boldsymbol{\xi}} \mathbf{x}\right)^{-t} \nabla_{\boldsymbol{\xi}}(g(h c+z))=\Pi,
$$

with the right-hand side

$$
\Pi \equiv-\left(\partial_{\xi} \mathbf{x}\right)^{-1}\left(\partial_{\xi \xi}^{2} \mathbf{x} \cdot V \cdot V\right)-\frac{1}{c^{2}}\left(\mathcal{V}^{t} \mathcal{H} \mathcal{V}\right)\left(\partial_{\boldsymbol{\xi}} \mathbf{x}\right)^{-1} \mathbf{s} .
$$

The number $g$ is the gravitation constant, and

$$
J_{0} \equiv \frac{1}{c} \operatorname{det}\left(\partial_{\boldsymbol{\xi}} \mathbf{x}\right)
$$

Equations (2.9), (2.10) can be combined to give a momentum equation in conservative form,

$$
\begin{aligned}
& \partial_{t}\left(J_{0} h V\right)+\nabla_{\boldsymbol{\xi}} \cdot\left(J_{0} h V V^{t}\right) \\
& +J_{0}\left(\partial_{\boldsymbol{\xi}} \mathbf{x}\right)^{-1}\left(\mathrm{Id}-\mathbf{s s}^{t}\right)\left(\partial_{\boldsymbol{\xi}} \mathbf{x}\right)^{-t}\left(\nabla_{\boldsymbol{\xi}}\left(\frac{1}{2} h^{2} g c\right)+h g \nabla_{\boldsymbol{\xi}} z+\frac{1}{2} g h^{2} \nabla_{\boldsymbol{\xi}} c\right) \\
& =J_{0} h \Pi .
\end{aligned}
$$

We say that this equation is in conservative form because differentiation of the unknowns $h, V$ appear only in conservative terms (the matrix factor in front of $\nabla_{\boldsymbol{\xi}}\left(\frac{1}{2} h^{2} g c\right)$ can be put under the $\nabla_{\boldsymbol{\xi}}$ symbol up to an additional term which contains no derivatives of $h$ and $V$ ).

Theorem 2.1. The system (2.9)-(2.10) has the properties

(i) it admits a conservative energy equation

$$
\begin{gathered}
\partial_{t}\left\{\frac{1}{2} J_{0} h\left[|\mathcal{V}|^{2}+\left(\mathbf{s}^{t} \mathcal{V} / c\right)^{2}\right]+\frac{1}{2} J_{0} h^{2} g c+J_{0} h g z\right\} \\
+\nabla_{\boldsymbol{\xi}} \cdot\left\{\left(\frac{1}{2}\left[|\mathcal{V}|^{2}+\left(\mathbf{s}^{t} \mathcal{V} / c\right)^{2}\right]+g(h c+z)\right) J_{0} h V\right\}=0,
\end{gathered}
$$

(ii) it preserves the steady state of a lake at rest $V=0, h c+z=$ const,

(iii) denoting by $\epsilon$ the aspect ratio between the width of the layer and the typical length of phenomena in $x$, the system is an approximation up to error terms in $\epsilon^{3}$ in (2.9) and in $\epsilon^{2}$ in (2.10) (or $\epsilon^{3}$ in (2.13)), as $\epsilon \rightarrow 0$, of the free surface incompressible Euler equations, under the only assumption that the curvature is small, $\mathcal{H}=\mathcal{O}(\epsilon)$. In particular, the velocity dependency in the normal variable needs not be specified.

The precise meaning of statement (iii) and its proof is the subject of Subsection 3.3 , so here we only prove (i) and (ii).

Proof of Theorem 2.1 (i)/(ii). The statement (ii) is obvious since for functions independent of time satisfying $V=0$ and $h c+z=$ const, (2.9) and (2.10) are satisfied. In order to prove (i), we multiply (2.10) by $\partial_{\boldsymbol{\xi}} \mathrm{x}$ and use identity $V \cdot \nabla_{\boldsymbol{\xi}}=\mathcal{V} \cdot \nabla_{x}$ to obtain the equation

$$
\partial_{t} \mathcal{V}+\mathcal{V} \cdot \nabla_{x} \mathcal{V}+\left(\mathrm{Id}-\mathbf{s s}^{t}\right) \nabla_{x}(g(h c+z))=-\frac{1}{c^{2}}\left(\mathcal{V}^{t} \mathcal{H} \mathcal{V}\right) \mathbf{s}
$$

Next, we take the scalar product of $(2.15)$ with the vector $\mathbf{s} / c$. Noticing that $\mathcal{V}$. $\nabla_{x}\left(\mathbf{s}^{t} / c\right)=\mathcal{V} \cdot \nabla_{x}\left(\partial_{x} z\right)=\mathcal{V}^{t} \mathcal{H} / c^{3}$, we get

$$
\begin{gathered}
\partial_{t}\left(\mathbf{s}^{t} \mathcal{V} / c\right)+\mathcal{V} \cdot \nabla_{x}\left(\mathbf{s}^{t} \mathcal{V} / c\right)+c \mathbf{s}^{t} \nabla_{x}(g(h c+z)) \\
=\left(\mathcal{V} \cdot \nabla_{x}\left(\mathbf{s}^{t} / c\right)\right) \mathcal{V}-\frac{1}{c^{3}}\left(\mathcal{V}^{t} \mathcal{H} \mathcal{V}\right)|\mathbf{s}|^{2}=\frac{1}{c} \mathcal{V}^{t} \mathcal{H} \mathcal{V} .
\end{gathered}
$$


Then, we multiply (2.15) by $\mathcal{V}$, we multiply $(2.16)$ by $\mathbf{s}^{t} \mathcal{V} / c$, and adding the results, this yields

$$
\partial_{t}\left\{\frac{1}{2}\left[|\mathcal{V}|^{2}+\left(\mathbf{s}^{t} \mathcal{V} / c\right)^{2}\right]\right\}+\mathcal{V} \cdot \nabla_{x}\left\{\frac{1}{2}\left[|\mathcal{V}|^{2}+\left(\mathbf{s}^{t} \mathcal{V} / c\right)^{2}\right]+g(h c+z)\right\}=0 .
$$

Noticing again that $\mathcal{V} \cdot \nabla_{x}=V \cdot \nabla_{\boldsymbol{\xi}}$, we finally multiply (2.17) by $J_{0} h$ and (2.9) by $\frac{1}{2}\left[|\mathcal{V}|^{2}+\left(\mathbf{s}^{t} \mathcal{V} / c\right)^{2}\right]+g(h c+z)$, which by addition give the energy equation (2.14).

We remark in passing that (2.15) shows that the model is independent of the chosen coordinates $\boldsymbol{\xi}$, since $\boldsymbol{\xi}$ does not enter any longer in this equation. The continuity equation (2.9) can also be formulated in a $\boldsymbol{\xi}$-independent manner, because according to the divergence chain rule, we have

$\nabla_{\boldsymbol{\xi}} \cdot\left(J_{0} h V\right)=\operatorname{det}\left(\partial_{\boldsymbol{\xi}} \mathbf{x}\right) \nabla_{x} \cdot(h \mathcal{V} / c)$, thus (2.9) gives

$$
\partial_{t}(h / c)+\nabla_{x} \cdot(h \mathcal{V} / c)=0 .
$$

In this spirit, the momentum equation takes the form

$$
\begin{aligned}
& \partial_{t}(h \mathcal{V} / c)+\nabla_{x} \cdot\left(h \mathcal{V} \mathcal{V}^{t} / c\right) \\
& +\frac{1}{c}\left(\mathrm{Id}-\mathbf{s s}^{t}\right)\left(\nabla_{x}\left(\frac{1}{2} h^{2} g c\right)+h g \nabla_{x} z+\frac{1}{2} g h^{2} \nabla_{x} c\right) \\
& =-\frac{h}{c^{3}}\left(\mathcal{V}^{t} \mathcal{H} \mathcal{V}\right) \mathbf{s} .
\end{aligned}
$$

Concerning weak solutions, i.e., possibly discontinuous solutions $h$ and $V$, we remark that as long as $z(x)$ and the change of coordinates $\mathbf{x}(\boldsymbol{\xi})$ are smooth, the formulations (2.9), (2.13) and (2.18), (2.19) both make sense (recall that the matrix factors in front of the pressure terms can be put inside the differentiation, up to an additional right-hand side). They are indeed equivalent, even for weak solutions, because they are obtained by multiplication by a smooth function. New equations obtained by the same procedure, such as the conservative version of $(2.26),(2.27)$ below, are also equivalent to the previous ones. However, for weak solutions, energy equations have to be changed into inequalities.

We would like now to make some remarks on the very special form of (2.10), or equivalently (2.15). The curvature term $\mathcal{V}^{t} \mathcal{H} \mathcal{V}$ that appears above can indeed be interpreted in terms of $\vec{n}$, as follows. Using (2.2), (2.3) and (2.4), we compute

$$
\begin{gathered}
\mathcal{V} \cdot \nabla_{x} \mathbf{s}=\left(\partial_{x} \mathbf{s}\right) \mathcal{V}=\frac{1}{c^{2}}\left(\mathrm{Id}-\mathbf{s s}^{t}\right) \mathcal{H} \mathcal{V} \\
\mathcal{V} \cdot \nabla_{x} c=-\frac{1}{c} \mathbf{s}^{t} \mathcal{V} \cdot \nabla_{x} \mathbf{s}=-\frac{1}{c} \mathbf{s}^{t} \mathcal{H} \mathcal{V} \\
\mathcal{V} \cdot \nabla_{x} \vec{n}=-\frac{1}{c^{2}}\left(\begin{array}{c}
\operatorname{Id}-\mathbf{s s}^{t} \\
c \mathbf{s}^{t}
\end{array}\right) \mathcal{H} \mathcal{V}
\end{gathered}
$$

and thus we get the relation

$$
\left(\mathcal{V} \cdot \nabla_{x} \vec{n}\right) \cdot \mathcal{V}^{t g}=-\frac{1}{c^{2}} \mathcal{V}^{t} \mathcal{H} \mathcal{V}
$$

Therefore, the right-hand side of (2.15) can be somehow better understood if we write the equation satisfied by $\mathcal{V}^{t g}$, which is obtained by putting (2.15) and (2.16) in a single 
$N+1$-dimensional equation,

$$
\begin{aligned}
\partial_{t} \mathcal{V}^{t g} & +\mathcal{V} \cdot \nabla_{x} \mathcal{V}^{t g}+\left(\begin{array}{c}
\mathrm{Id}-\mathbf{s} \mathbf{s}^{t} \\
c \mathbf{s}^{t}
\end{array}\right) \nabla_{x}(g(h c+z)) \\
& =\frac{1}{c^{2}}\left(\mathcal{V}^{t} \mathcal{H} \mathcal{V}\right) \vec{n} \\
& =-\left(\left(\mathcal{V} \cdot \nabla_{x} \vec{n}\right) \cdot \mathcal{V}^{t g}\right) \vec{n} .
\end{aligned}
$$

In this equation, the right-hand side has the role to preserve the condition $\mathcal{V}^{t g} \cdot \vec{n}=0$. If we neglect the gravity, it only remains $\partial_{t} \mathcal{V}^{t g}+\mathcal{V} \cdot \nabla_{x} \mathcal{V}^{t g}=-\left(\left(\mathcal{V} \cdot \nabla_{x} \vec{n}\right) \cdot \mathcal{V}^{t g}\right) \vec{n}$, and the physical interpretation of this equation is that the particles are advected with the only constraint to remain on the surface $\mathcal{S}$.

In order to make a transition to our more general model without the small curvature assumption, which we will present in the next subsection, let us now write another equivalent formulation of the model (2.9), (2.10). It uses a new velocity parametrization $W(t, \boldsymbol{\xi}) \in \mathbb{R}^{N}$, linked to $V$ and to $\mathcal{V}=\left(\partial_{\boldsymbol{\xi}} \mathbf{x}\right) V$ by

$$
W \equiv M_{0}^{-1} V=\left(\partial_{\boldsymbol{\xi}} \mathbf{x}\right)^{t}\left(\mathrm{Id}+\mathbf{s s}^{t} / c^{2}\right) \mathcal{V},
$$

with matrix $M_{0}$ defined by

$$
M_{0} \equiv\left(\partial_{\boldsymbol{\xi}} \mathbf{x}\right)^{-1}\left(\mathrm{Id}-\mathbf{s} \mathbf{s}^{t}\right)\left(\partial_{\boldsymbol{\xi}} \mathbf{x}\right)^{-t}
$$

The relation with the material velocity $\mathcal{V}^{t g}$ is still given by (2.8).

Proposition 2.2. The system (2.9)-(2.10) is equivalent to

$$
\begin{gathered}
\partial_{t}\left(J_{0} h\right)+\nabla_{\boldsymbol{\xi}} \cdot\left(J_{0} h M_{0} W\right)=0 \\
\partial_{t} W+\left(\partial_{\boldsymbol{\xi}} W-\left(\partial_{\boldsymbol{\xi}} W\right)^{t}\right) M_{0} W+\nabla_{\boldsymbol{\xi}}\left(\frac{1}{2} W^{t} M_{0} W+g(h c+z)\right)=0 .
\end{gathered}
$$

Moreover, the energy equation (2.14) takes the form

$$
\begin{gathered}
\partial_{t}\left\{\frac{1}{2} J_{0} h W^{t} M_{0} W+\frac{1}{2} J_{0} h^{2} g c+J_{0} h g z\right\} \\
+\nabla_{\boldsymbol{\xi}} \cdot\left\{\left(\frac{1}{2} W^{t} M_{0} W+g(h c+z)\right) J_{0} h M_{0} W\right\}=0 .
\end{gathered}
$$

Proof. The first equation (2.26) is obviously the same as (2.9) since $V=M_{0} W$. Then we compute from $(2.24)$

$$
W^{t} M_{0} W=\mathcal{V}^{t}\left(\operatorname{Id}+\mathbf{s s}^{t} / c^{2}\right) \mathcal{V}=|\mathcal{V}|^{2}+\left(\mathbf{s}^{t} \mathcal{V} / c\right)^{2}=\left|\mathcal{V}^{t g}\right|^{2}
$$

and (2.28) follows. The velocity equation is more involved. We multiply $(2.16)$ by $\mathbf{s} / c$ and add it to (2.15). We get

$$
\begin{aligned}
& \partial_{t}\left(\left(\operatorname{Id}+\mathbf{s} \mathbf{s}^{t} / c^{2}\right) \mathcal{V}\right)+\mathcal{V} \cdot \nabla_{x}\left(\left(\operatorname{Id}+\mathbf{s} \mathbf{s}^{t} / c^{2}\right) \mathcal{V}\right)+\nabla_{x}(g(h c+z)) \\
= & \left(\mathcal{V} \cdot \nabla_{x}(\mathbf{s} / c)\right) \mathbf{s}^{t} \mathcal{V} / c \equiv B .
\end{aligned}
$$


But since $\partial_{x}\left(\mathbf{s}^{t} / c\right)=\partial_{x x}^{2} z$ is symmetric, the $j$ th component of the right-hand side is

$$
\begin{aligned}
B_{j} & =\left(\mathcal{V} \cdot \nabla_{x}\left(\mathbf{s}_{j} / c\right)\right) \mathbf{s}^{t} \mathcal{V} / c \\
& =\left(\mathcal{V}^{t} \partial_{x_{j}}(\mathbf{s} / c)\right) \mathbf{s}^{t} \mathcal{V} / c=\frac{1}{2} \mathcal{V}^{t} \partial_{x_{j}}\left(\mathbf{s s}^{t} / c^{2}\right) \mathcal{V} .
\end{aligned}
$$

So by definitions $(2.24),(2.25)$ we can write

$$
\begin{aligned}
B_{j}= & \frac{1}{2} W^{t}\left(\partial_{\boldsymbol{\xi}} \mathbf{x}\right)^{-1}\left(\operatorname{Id}-\mathbf{s s}^{t}\right) \partial_{x_{j}}\left(\operatorname{Id}+\mathbf{s s}^{t} / c^{2}\right)\left(\operatorname{Id}-\mathbf{s s}^{t}\right)\left(\partial_{\boldsymbol{\xi}} \mathbf{x}\right)^{-t} W \\
= & -\frac{1}{2} W^{t}\left(\partial_{\boldsymbol{\xi}} \mathbf{x}\right)^{-1} \partial_{x_{j}}\left(\operatorname{Id}-\mathbf{s} \mathbf{s}^{t}\right)\left(\partial_{\boldsymbol{\xi}} \mathbf{x}\right)^{-t} W \\
= & -\frac{1}{2} W^{t} \partial_{x_{j}}\left(\left(\partial_{\boldsymbol{\xi}} \mathbf{x}\right)^{-1}\left(\operatorname{Id}-\mathbf{s s}^{t}\right)\left(\partial_{\boldsymbol{\xi}} \mathbf{x}\right)^{-t}\right) W \\
& +W^{t}\left(\partial_{x_{j}}\left(\partial_{\boldsymbol{\xi}} \mathbf{x}\right)^{-1}\right)\left(\operatorname{Id}-\mathbf{s} \mathbf{s}^{t}\right)\left(\partial_{\boldsymbol{\xi}} \mathbf{x}\right)^{-t} W \\
= & -\frac{1}{2} W^{t}\left(\partial_{x_{j}} M_{0}\right) W-W^{t}\left(\left(\partial_{\boldsymbol{\xi}} \mathbf{x}\right)^{-1}\left(\partial_{x_{j}}\left(\partial_{\boldsymbol{\xi}} \mathbf{x}\right)\right)\left(\partial_{\boldsymbol{\xi}} \mathbf{x}\right)^{-1}\right) \mathcal{V} .
\end{aligned}
$$

Therefore,

$$
\begin{aligned}
\left(\left(\partial_{\xi} \mathbf{x}\right)^{t} B\right)_{i} & =-\frac{1}{2} W^{t}\left(\partial_{\boldsymbol{\xi}_{i}} M_{0}\right) W-W^{t}\left(\partial_{\boldsymbol{\xi}} \mathbf{x}\right)^{-1}\left(\partial_{\boldsymbol{\xi}_{i}}\left(\partial_{\boldsymbol{\xi}} \mathbf{x}\right)\right)\left(\partial_{\boldsymbol{\xi}} \mathbf{x}\right)^{-1} \mathcal{V} \\
& =-\frac{1}{2} W^{t}\left(\partial_{\boldsymbol{\xi}_{i}} M_{0}\right) W-W^{t}\left(\partial_{\boldsymbol{\xi}} \mathbf{x}\right)^{-1}\left(\partial_{x}\left(\partial_{\boldsymbol{\xi}_{i}} \mathbf{x}\right)\right) \mathcal{V} \\
& =-\frac{1}{2} W^{t}\left(\partial_{\boldsymbol{\xi}_{i}} M_{0}\right) W-\left(\mathcal{V} \cdot \nabla_{x}\left(\partial_{\boldsymbol{\xi}_{i}} \mathbf{x}\right)\right) \cdot\left(\partial_{\boldsymbol{\xi}} \mathbf{x}\right)^{-t} W,
\end{aligned}
$$

and defining $K_{i}=W^{t}\left(\partial_{\boldsymbol{\xi}_{i}} M_{0}\right) W$, this yields

$$
\left(\partial_{\boldsymbol{\xi}} \mathbf{x}\right)^{t} B=\frac{1}{2} K-\left(\mathcal{V} \cdot \nabla_{x}\left(\partial_{\boldsymbol{\xi}} \mathbf{x}\right)^{t}\right) \cdot\left(\partial_{\boldsymbol{\xi}} \mathbf{x}\right)^{-t} W .
$$

Finally, we multiply $(2.30)$ by $\left(\partial_{\boldsymbol{\xi}} \mathbf{x}\right)^{t}$ and obtain

$$
\partial_{t} W+\mathcal{V} \cdot \nabla_{x} W+\nabla_{\boldsymbol{\xi}}(g(h c+z))=-\frac{1}{2} K .
$$

Using the fact that $\mathcal{V} \cdot \nabla_{x}=V \cdot \nabla_{\boldsymbol{\xi}}$ and $V=M_{0} W$, this gives $(2.27)$.

A striking property of the formulation (2.27) is that it implies that $\operatorname{curl}_{\boldsymbol{\xi}} W \equiv \partial_{\boldsymbol{\xi}} W-\left(\partial_{\boldsymbol{\xi}} W\right)^{t}$ is transported by $V=M_{0} W$.

\subsection{A multidimensional shallow water model for arbitrary topography.}

This model is described by

$$
h(t, \boldsymbol{\xi}) \geq 0, \quad W(t, \boldsymbol{\xi}) \in \mathbb{R}^{N},
$$

where as before $h$ is the width of the material layer in direction normal to the topography, and $W$ is a parametrization of the velocity field that is linked to the $N+1-$ dimensional material velocity vector $\mathcal{V}^{t g}$ by

$$
\mathcal{V}^{t g}=\left(\begin{array}{c}
\mathrm{Id} \\
\frac{1}{c} \mathbf{s}^{t}
\end{array}\right)\left(\mathrm{Id}-\mathbf{s s}^{t}\right)\left(\mathrm{Id}-\frac{1}{2} h \partial_{x} \mathbf{s}\right)^{-t}\left(\partial_{\boldsymbol{\xi}} \mathbf{x}\right)^{-t} W .
$$

Notice that this relation is now time dependent, via the function $h(t, \boldsymbol{\xi})$. The model reads as

$$
\partial_{t} \int_{0}^{h} J d \bar{\xi}+\nabla_{\boldsymbol{\xi}} \cdot\left(\int_{0}^{h} J M d \bar{\xi} W\right)=0
$$




$$
\partial_{t} W+\left(\partial_{\boldsymbol{\xi}} W-\left(\partial_{\boldsymbol{\xi}} W\right)^{t}\right) \frac{\int_{0}^{h} J M d \bar{\xi}}{\int_{0}^{h} J d \bar{\xi}} W+\nabla_{\boldsymbol{\xi}}\left(\frac{1}{2} W^{t} M_{h} W+g(h c+z)\right)=0
$$

where $M(\boldsymbol{\xi}, \bar{\xi})$ and $J(\boldsymbol{\xi}, \bar{\xi})$ are defined for $\bar{\xi} \geq 0$ small enough by

$$
\begin{gathered}
M \equiv\left(\partial_{\boldsymbol{\xi}} \mathbf{x}\right)^{-1}\left(\operatorname{Id}-\bar{\xi} \partial_{x} \mathbf{s}\right)^{-1}\left(\operatorname{Id}-\mathbf{s} \mathbf{s}^{t}\right)\left(\operatorname{Id}-\bar{\xi} \partial_{x} \mathbf{s}\right)^{-t}\left(\partial_{\boldsymbol{\xi}} \mathbf{x}\right)^{-t}, \\
J \equiv(\operatorname{det} M)^{-1 / 2}=\frac{1}{c} \operatorname{det}\left(\left(\operatorname{Id}-\bar{\xi} \partial_{x} \mathbf{s}\right) \partial_{\boldsymbol{\xi}} \mathbf{x}\right),
\end{gathered}
$$

and

$$
J_{h}(t, \boldsymbol{\xi}) \equiv J(\boldsymbol{\xi}, \bar{\xi}=h(t, \boldsymbol{\xi})), \quad M_{h}(t, \boldsymbol{\xi}) \equiv M(\boldsymbol{\xi}, \bar{\xi}=h(t, \boldsymbol{\xi})) .
$$

Notice that with these notations, $J_{0}$ and $M_{0}$ defined previously in equations (2.12) and (2.25) can be understood as $J_{0}(\boldsymbol{\xi})=J(\boldsymbol{\xi}, \bar{\xi}=0)$ and $M_{0}(\boldsymbol{\xi})=M(\boldsymbol{\xi}, \bar{\xi}=0)$. The velocity equation (2.36) has the advantage of making apparent the transport equation on $\operatorname{curl}_{\boldsymbol{\xi}} W=\partial_{\boldsymbol{\xi}} W-\left(\partial_{\boldsymbol{\xi}} W\right)^{t}$, but it can be replaced by the momentum equation

$$
\begin{aligned}
& \partial_{t}\left(\int_{0}^{h} J d \bar{\xi} W\right)+\nabla_{\boldsymbol{\xi}} \cdot\left(W \otimes \int_{0}^{h} J M d \bar{\xi} W\right)-\left(\partial_{\boldsymbol{\xi}} W\right)^{t} \int_{0}^{h} J M d \bar{\xi} W \\
& +\left(\int_{0}^{h} J d \bar{\xi}\right) \nabla_{\boldsymbol{\xi}}\left(\frac{1}{2} W^{t} M_{h} W+g(h c+z)\right)=0
\end{aligned}
$$

or in conservative form

$$
\begin{aligned}
& \partial_{t}\left(\int_{0}^{h} J d \bar{\xi} W\right)+\nabla_{\boldsymbol{\xi}} \cdot\left(W \otimes \int_{0}^{h} J M d \bar{\xi} W\right) \\
& +\nabla_{\boldsymbol{\xi}}\left\{\int_{0}^{h} J d \bar{\xi} \frac{1}{2} W^{t} M_{h} W-\frac{1}{2} W^{t} \int_{0}^{h} J M d \bar{\xi} W+\int_{0}^{h} J d \bar{\xi} g(h c+z)\right\} \\
& -J_{h} g(h c+z) \nabla_{\boldsymbol{\xi}} h \\
& =\int_{0}^{h} \nabla_{\boldsymbol{\xi}} J d \bar{\xi}\left(\frac{1}{2} W^{t} M_{h} W+g(h c+z)\right)-\frac{1}{2} W^{t} \int_{0}^{h} \nabla_{\boldsymbol{\xi}}(J M) d \bar{\xi} W
\end{aligned}
$$

Indeed we have for the term in $\nabla_{\xi} h$

$$
J_{h} g(h c+z) \nabla_{\boldsymbol{\xi}} h=\nabla_{\boldsymbol{\xi}}\left(\int_{0}^{h} J g(\bar{\xi} c+z) d \bar{\xi}\right)-\int_{0}^{h} \nabla_{\boldsymbol{\xi}}(J g(\bar{\xi} c+z)) d \bar{\xi} .
$$

Theorem 2.3. The system (2.35)-(2.36) has the properties (i) it admits a conservative energy equation

$$
\begin{array}{r}
\partial_{t}\left\{\frac{1}{2} W^{t} \int_{0}^{h} J M d \bar{\xi} W+g c \int_{0}^{h} J \bar{\xi} d \bar{\xi}+g z \int_{0}^{h} J d \bar{\xi}\right\} \\
+\nabla_{\boldsymbol{\xi}} \cdot\left\{\left(\frac{1}{2} W^{t} M_{h} W+g(h c+z)\right) \int_{0}^{h} J M d \bar{\xi} W\right\}=0,
\end{array}
$$


(ii) it preserves the steady state of a lake at rest $W=0, h c+z=$ const,

(iii) denoting by $\epsilon$ the aspect ratio between the width of the layer and the typical length of phenomena in $x$, the system is an approximation up to error terms in $\epsilon^{3}$ in (2.35) and in $\epsilon^{2}$ in (2.36) (or $\epsilon^{3}$ in (2.41)), as $\epsilon \rightarrow 0$, of the free surface incompressible Euler equations, without any assumption on the topography nor on the velocity profile in the normal variable.

The precise meaning of statement (iii) and its proof is the subject of Subsection 3.4 , and since (ii) is obvious, we only prove here (i).

Proof of Theorem 2.3 (i). Multiply (2.36) by $\int_{0}^{h} J M d \bar{\xi} W,(2.35)$ by $\frac{1}{2} W^{t} M_{h} W+$ $g(h c+z)$ and add up the results. It gives

$$
\begin{aligned}
& \left(\frac{1}{2} W^{t} M_{h} W+g(h c+z)\right) J_{h} \partial_{t} h+\partial_{t} W^{t}\left(\int_{0}^{h} J M d \bar{\xi} W\right) \\
& +\nabla_{\boldsymbol{\xi}} \cdot\left\{\left(\frac{1}{2} W^{t} M_{h} W+g(h c+z)\right) \int_{0}^{h} J M d \bar{\xi} W\right\}=0,
\end{aligned}
$$

leading to (2.43).

In the case of small curvature $\mathcal{H}=\mathcal{O}(\epsilon)$, we have $\partial_{x} \mathbf{s}=\mathcal{O}(\epsilon)$, and since by definition of $\epsilon$ we have $h=\mathcal{O}(\epsilon)$, we deduce that for $0 \leq \bar{\xi} \leq h, M=M_{0}+\mathcal{O}\left(\epsilon^{2}\right)$, $J=J_{0}+\mathcal{O}\left(\epsilon^{2}\right)$, thus $(2.35)-(2.36)$ reduces to $(2.26)-(2.27)$ up to terms in $\epsilon^{3}$ and $\epsilon^{2}$, respectively.

2.4. Coulomb friction. A bottom friction term of the type described in [5] can be included in either the model for small slope variation of Subsection 2.2 or the model for arbitrary topography of Subsection 2.3.

2.4.1. Friction with small curvature. In the case of model (2.9)-(2.10), an extra term has to be introduced as

$$
\begin{gathered}
\partial_{t}\left(J_{0} h\right)+\nabla_{\boldsymbol{\xi}} \cdot\left(J_{0} h V\right)=0, \\
\partial_{t} V+V \cdot \nabla_{\boldsymbol{\xi}} V+\left(\partial_{\boldsymbol{\xi}} \mathbf{x}\right)^{-1}\left(\mathrm{Id}-\mathbf{s s}^{t}\right)\left(\partial_{\boldsymbol{\xi}} \mathbf{x}\right)^{-t} \nabla_{\boldsymbol{\xi}}(g(h c+z)) \\
=\Pi-\frac{g \mu c V}{\sqrt{|\mathcal{V}|^{2}+\left(\mathbf{s}^{t} \mathcal{V} / c\right)^{2}}}\left(1+\frac{\mathcal{V}^{t} \mathcal{H} \mathcal{V}}{g c^{3}}\right)_{+},
\end{gathered}
$$

where $\Pi$ is still given by (2.11), and with the same notations. The denominator in the friction term is nothing else than the norm of the material velocity $\mathcal{V}^{t g}$ in (2.8),

$$
|\mathcal{V}|^{2}+\left(\mathbf{s}^{t} \mathcal{V} / c\right)^{2}=\mathcal{V}^{t}\left(\operatorname{Id}+\mathbf{s s}^{t} / c^{2}\right) \mathcal{V}=\left|\mathcal{V}^{t g}\right|^{2}
$$

The friction coefficient $\mu \geq 0$ could be any function of $t$ and $\boldsymbol{\xi}$, but for physical relevance it should depend only on $h$ and $\left|\mathcal{V}^{t g}\right|$, see [9]. The scalar $\mathcal{V}^{t} \mathcal{H} \mathcal{V}$ is the curvature of the topography in the direction of the flow. It can be positive or negative according to the local convexity or concavity of the surface. The index + stands for the positive part, $x_{+}=\max (0, x)$. It appears here because when the expression between parentheses becomes negative, the material should leave the topographical surface, giving thus a vanishing (instead of negative) friction. 
In the horizontal coordinate formulation the model reads as

$$
\begin{gathered}
\partial_{t}(h / c)+\nabla_{x} \cdot(h \mathcal{V} / c)=0 \\
\partial_{t} \mathcal{V}+\mathcal{V} \cdot \nabla_{x} \mathcal{V}+\left(\operatorname{Id}-\mathbf{s s}^{t}\right) \nabla_{x}(g(h c+z)) \\
=-\frac{1}{c^{2}}\left(\mathcal{V}^{t} \mathcal{H} \mathcal{V}\right) \mathbf{s}-\frac{g \mu c \mathcal{V}}{\sqrt{|\mathcal{V}|^{2}+\left(\mathbf{s}^{t} \mathcal{V} / c\right)^{2}}}\left(1+\frac{\mathcal{V}^{t} \mathcal{H} \mathcal{V}}{g c^{3}}\right)_{+},
\end{gathered}
$$

see (2.18), (2.15). In (2.45)-(2.46) or (2.48)-(2.49), we have to make precise the meaning of the friction term when $V=0$, or equivalently when $\mathcal{V}=0$. Indeed we have to understand the ratio $\mathcal{R}=\mathcal{V} /\left|\mathcal{V}^{t g}\right|$ as multivalued. This means that when $\mathcal{V}=0$, $\mathcal{R}$ can take any value that can be obtained from a limit when $\mathcal{V} \rightarrow 0$, i.e., $\mathcal{R}$ can be any vector such that $|\mathcal{R}|^{2}+\left(\mathbf{s}^{t} \mathcal{R} / c\right)^{2} \equiv \mathcal{R}^{t}\left(\operatorname{Id}+\mathbf{s s}^{t} / c^{2}\right) \mathcal{R} \leq 1$, or equivalently $\left|\mathcal{R}^{t g}\right| \leq 1$, by adopting the abstract notation $\mathcal{R}^{t g} \equiv\left(\mathcal{R}, \mathbf{s}^{t} \mathcal{R} / c\right)$. With this interpretation, one can check easily that the friction term dissipates energy, in the sense that the righthand side that enters into the energy identity (2.14) is always nonpositive. This interpretation also gives directly the steady states of (2.48)-(2.49), which are those for which

$$
\mathcal{V}=0, \quad\left|\left[\left(\operatorname{Id}-\mathbf{s s}^{t}\right) \nabla_{x}(h c+z)\right]^{t g}\right| \leq \mu c
$$

or more explicitly

$$
\mathcal{V}=0, \quad\left(\nabla_{x}(h c+z)^{t}\right)\left(\operatorname{Id}-\mathbf{s s}^{t}\right) \nabla_{x}(h c+z) \leq \mu^{2} c^{2} .
$$

A rigorous existence result has been proved for such a multivalued friction term in [6].

Another velocity parametrization of interest is

$$
u=\mathcal{V} / c,
$$

for which the relation with the material velocity $\mathcal{V}^{t g}$ in (2.8) becomes

$$
\mathcal{V}^{t g}=(c u, \mathbf{s} \cdot u) .
$$

Note that this is close to a rotation of $u$ since $|\mathbf{s}|^{2}+c^{2}=1$. In dimension $N=1, u$ is effectively the scalar physical velocity in the direction of the topography. The system satisfied by $h$ and $u$ is

$$
\begin{gathered}
\partial_{t}(h / c)+\nabla_{x} \cdot(h u)=0 \\
\partial_{t} u+c u \cdot \nabla_{x} u+\frac{1}{c}\left(\mathrm{Id}-\mathbf{s s}^{t}\right) \nabla_{x}(g(h c+z)) \\
=-\frac{1}{c}\left(u^{t} \mathcal{H} u\right) \mathbf{s}+\frac{1}{c}\left(\mathbf{s}^{t} \mathcal{H} u\right) u-\frac{g \mu c u}{\sqrt{c^{2}|u|^{2}+(\mathbf{s} \cdot u)^{2}}}\left(1+\frac{u^{t} \mathcal{H} u}{g c}\right)_{+} .
\end{gathered}
$$

The model can also be written with the normal variable $W$. The system (2.26)-(2.27) is then modified as

$$
\partial_{t}\left(J_{0} h\right)+\nabla_{\boldsymbol{\xi}} \cdot\left(J_{0} h M_{0} W\right)=0,
$$




$$
\begin{aligned}
& \partial_{t} W+\left(\partial_{\boldsymbol{\xi}} W-\left(\partial_{\boldsymbol{\xi}} W\right)^{t}\right) M_{0} W+\nabla_{\boldsymbol{\xi}}\left(\frac{1}{2} W^{t} M_{0} W+g(h c+z)\right) \\
=- & \frac{g \mu c W}{\sqrt{W^{t} M_{0} W}}\left(1+\frac{\mathcal{V}^{t} \mathcal{H} \mathcal{V}}{g c^{3}}\right)_{+},
\end{aligned}
$$

with the relation (2.24) linking $\mathcal{V}$ to $W$, that gives $\left|\mathcal{V}^{t g}\right|^{2}=W^{t} M_{0} W$.

Theorem 2.4. The system (2.45)-(2.46) (or equivalently (2.48)-(2.49) or (2.54)(2.55) or (2.56)-(2.57)) is an approximation, up to errors in $\mathcal{O}\left(\epsilon^{3}\right)$ and $\mathcal{O}\left(\epsilon^{2}\right)$ respectively for mass and velocity equations, as the aspect ratio $\epsilon \rightarrow 0$, of the free surface incompressible Navier-Stokes equations with viscosity $\nu$ and Coulomb bottom friction with coefficient $\mu$, under the assumptions that $\nu=o\left(\epsilon^{2}\right), \mu=\mathcal{O}(\nu / \epsilon)$, and the curvature is small $\mathcal{H}=\mathcal{O}(\epsilon)$.

More precise statements are provided within the proof of this theorem, which is given in Subsection 3.5. Notice that with the assumption of smallness of the friction $\mu$, the curvature term leads to a correction of order $\epsilon^{2}$ that could just be removed. However, we keep it in order to have a more accurate model.

Notice also that with our assumption $\nu=o\left(\epsilon^{2}\right)$, the boundary layer induced by viscosity, which is of order $\sqrt{\nu t}$, is much smaller than the material layer, which is of order $\epsilon$. This situation is very different from that considered in [3] where $\nu \sim \epsilon$, which implies that the lengthscale induced by the viscosity is much larger than $\epsilon$.

2.4.2. Friction with arbitrary topography. The model for arbitrary topography (2.35)-(2.36) has to include a friction term of the form

$$
\begin{gathered}
\partial_{t} \int_{0}^{h} J d \bar{\xi}+\nabla_{\boldsymbol{\xi}} \cdot\left(\int_{0}^{h} J M d \bar{\xi} W\right)=0 \\
\partial_{t} W+\left(\partial_{\boldsymbol{\xi}} W-\left(\partial_{\boldsymbol{\xi}} W\right)^{t}\right) \frac{\int_{0}^{h} J M d \bar{\xi}}{\int_{0}^{h} J d \bar{\xi}} W+\nabla_{\boldsymbol{\xi}}\left(\frac{1}{2} W^{t} M_{h} W+g(h c+z)\right) \\
=-J_{0} \frac{\mu W}{\sqrt{W^{t} M_{0} W}} \frac{\left(g h c+\frac{1}{2} W^{t}\left(M_{h}-M_{0}\right) W\right)_{+}}{\int_{0}^{h} J d \bar{\xi}} .
\end{gathered}
$$

Theorem 2.5. In the regime $\operatorname{curl} W=\mathcal{O}(\epsilon)$, the system (2.58)-(2.59) is an approximation, up to errors in $\mathcal{O}\left(\epsilon^{3}\right)$ and $\mathcal{O}\left(\epsilon^{2}\right)$ respectively for mass and velocity equations, as the aspect ratio $\epsilon \rightarrow 0$, of the free surface incompressible Navier-Stokes equations with viscosity $\nu$ and Coulomb bottom friction with coefficient $\mu$, under the assumptions that $\nu=o\left(\epsilon^{2}\right)$ and $\mu=\mathcal{O}(\nu / \epsilon)$.

The proof of this theorem is provided in Subsection 3.5.

\section{Derivation of the models}

In this section, we derive the models introduced in Section 2 from the incompressible Euler equations with free boundary.

Notation. In the following, a vector in $\mathbb{R}^{N+1}$ is marked by an arrow. The first components of it which form a vector in $\mathbb{R}^{N}$ are denoted by bold symbols, the last component by an overbar, as in $\vec{X}=(\mathbf{X}, \bar{X})$. If symbols have an arrow, then indices are running from 1 to $N+1$. For bold symbols, indices range from 1 to $N$. 


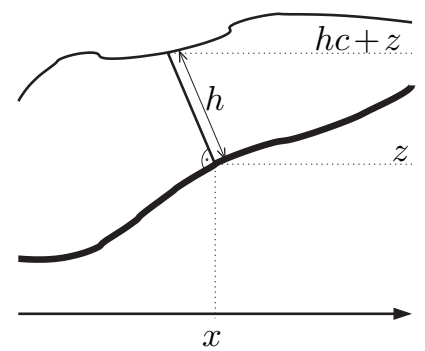

FIG. 3.1. Material layer above the graph of $z$.

3.1. Geometry of the layer. Consider a topography $\mathcal{S}$, as described in Subsection 2.1, and assume a parametrization of $\mathcal{S}$ is given, via an invertible map $\boldsymbol{\xi} \mapsto$ $\mathbf{x}(\boldsymbol{\xi})$ for $\boldsymbol{\xi} \in \mathbb{R}^{N}$. Then we construct a coordinate system in a suitable neighboorhood above the graph of $z$ as follows. We define a map $\vec{\xi} \mapsto \vec{X}(\vec{\xi})$ for $\vec{\xi}=(\boldsymbol{\xi}, \bar{\xi}) \in \mathbb{R}^{N+1}, \bar{\xi}>0$, by

$$
\vec{X}(\vec{\xi}) \equiv(\mathbf{X}, \bar{X})=\left(\begin{array}{c}
\mathbf{x}(\boldsymbol{\xi}) \\
z(\mathbf{x}(\boldsymbol{\xi}))
\end{array}\right)+\bar{\xi}\left(\begin{array}{c}
-\mathbf{s}(\mathbf{x}(\boldsymbol{\xi})) \\
c(\mathbf{x}(\boldsymbol{\xi}))
\end{array}\right) .
$$

Note that the second vector on the right-hand side is just the normal $\vec{n}$, written as a function of $\boldsymbol{\xi}$. The mapping (3.1) is (locally) invertible whenever $\bar{\xi}$ is small enough, so that the Jacobian has full rank. This defines an open neighboorhood above the graph of $z$. We are going to describe a material layer filling the domain

$$
\Omega_{t} \equiv\left\{\vec{X}(\vec{\xi}) \in \mathbb{R}^{N+1} \mid 0<\bar{\xi}<h(t, \boldsymbol{\xi})\right\},
$$

see Figure 3.1, thus we assume that $\vec{X}$ is a diffeomorphism in $\Omega_{t}$.

The Jacobian matrix of (3.1) defines the matrix $A$ by

$$
\begin{gathered}
A^{-1}=\partial_{\vec{\xi}} \vec{X}=\left(\begin{array}{cc}
\mathrm{Id} & -\mathbf{s} \\
\frac{1}{c} \mathbf{s}^{t} & c
\end{array}\right)\left(\begin{array}{cc}
\partial_{\boldsymbol{\xi}} \mathbf{X} & 0 \\
0 & 1
\end{array}\right), \\
A=\left(\partial_{\vec{\xi}} \vec{X}\right)^{-1}=\left(\begin{array}{cc}
\left(\partial_{\boldsymbol{\xi}} \mathbf{X}\right)^{-1} & 0 \\
0 & 1
\end{array}\right)\left(\begin{array}{cc}
\mathrm{Id}-\mathbf{s s}^{t} & c \mathbf{s} \\
-\mathbf{s}^{t} & c
\end{array}\right),
\end{gathered}
$$

with

$$
\partial_{\boldsymbol{\xi}} \mathbf{X}=\left(\operatorname{Id}-\bar{\xi} \partial_{x} \mathbf{s}\right) \partial_{\boldsymbol{\xi}} \mathbf{x}
$$

For further reference, we compute

$$
A A^{t}=\left(\begin{array}{cc}
M & 0 \\
0 & 1
\end{array}\right) \quad \text { with } \quad M=\left(\partial_{\boldsymbol{\xi}} \mathbf{X}\right)^{-1}\left(\mathrm{Id}-\mathbf{s s}^{t}\right)\left(\partial_{\boldsymbol{\xi}} \mathbf{X}\right)^{-t}
$$

The determinant of (3.3) is given by

$$
J \equiv \operatorname{det}\left(\partial_{\vec{\xi}} \vec{X}\right)=\frac{1}{c} \operatorname{det}\left(\partial_{\boldsymbol{\xi}} \mathbf{X}\right)=(\operatorname{det} M)^{-1 / 2},
$$

and the differential operators transform according to

$$
\nabla_{\vec{X}}=A^{t} \nabla_{\vec{\xi}}
$$


3.2. Transformation of Euler equations. We start from the incompressible Euler equations in cartesian coordinates. Assume that velocity and pressure fields

$$
\vec{U}(t, \vec{X}) \in \mathbb{R}^{N+1}, \quad P(t, \vec{X}) \in \mathbb{R}
$$

are given for $t>0$ and $\vec{X} \in \Omega_{t}$, that satisfy

$$
\begin{aligned}
\partial_{t} \vec{U}+\left(\vec{U} \cdot \nabla_{\vec{X}}\right) \vec{U}+\nabla_{\vec{X}} P & =-\vec{g}, \\
\nabla_{\vec{X}} \cdot \vec{U} & =0,
\end{aligned}
$$

where $\vec{g}=g\left(\begin{array}{l}0 \\ 1\end{array}\right)$, and the following boundary conditions: at the bed

$$
\vec{U} \cdot \vec{n}=0 \quad \text { for } \quad \bar{\xi}=0
$$

and at the free surface

$$
P=0 \text { for } \bar{\xi}=h(t, \boldsymbol{\xi}) .
$$

We also have to give a rule for the evolution of the free surface:

The free surface is advected by the material velocity $\vec{U}$.

We are going to transform these Euler equations into the curvilinear coordinate system defined in Subsection 3.1. Moreover, starting from the horizontal and vertical velocity components $\vec{U}=(\mathbf{U}, \bar{U})$, we are going to decompose $\vec{U}$ into the part tangential to the topography and the normal part, and write the corresponding momentum equations. This can be done in several ways, and we have selected two important choices which lead to two different formulations, even if they are of course equivalent.

3.2.1. First velocity decomposition: by jacobian matrix. A natural way of choosing new velocity components is to define a parameter vector

$$
\vec{V} \equiv(\mathbf{V}, \bar{V})=A \vec{U} .
$$

According to (3.8), we then obtain

$$
\vec{U} \cdot \nabla_{\vec{X}}=\vec{V} \cdot \nabla_{\vec{\xi}} \cdot
$$

Note that (3.15) and (3.4) read

$$
\left(\partial_{\boldsymbol{\xi}} \mathbf{X}\right) \mathbf{V}=\mathbf{U}+\mathbf{s} \bar{V}, \quad \bar{V}=\vec{n} \cdot \vec{U},
$$

thus $\bar{V}$ is the normal component of $\vec{U}$. Using (3.3) we also find

$$
\mathbf{U}=\left(\partial_{\boldsymbol{\xi}} \mathbf{X}\right) \mathbf{V}-\mathbf{s} \bar{V}, \quad \bar{U}=\frac{1}{c} \mathbf{s}^{t}\left(\partial_{\boldsymbol{\xi}} \mathbf{X}\right) \mathbf{V}+c \bar{V} .
$$

This yields in particular the tangential part of $\vec{U}$,

$$
\vec{U}-(\vec{n} \cdot \vec{U}) \vec{n}=\left(\begin{array}{c}
\text { Id } \\
\frac{1}{c} \mathbf{s}^{t}
\end{array}\right)\left(\partial_{\boldsymbol{\xi}} \mathbf{X}\right) \mathbf{V} .
$$

A first equation is obtained by applying the divergence chain rule to the incompressibility condition (3.11),

$$
\nabla_{\vec{\xi}} \cdot(J \vec{V})=J \nabla_{\vec{X}} \cdot \vec{U}=0 .
$$


Observe that once $\mathbf{V}$ is known, this equation determines $\bar{V}$ in a unique way with the boundary condition (3.12). Next, to write down a momentum equation for $\vec{V}$, we multiply (3.10) by $A$, noticing that

$$
\vec{g}=\nabla_{\vec{X}}(\vec{g} \cdot \vec{X})=\nabla_{\vec{X}}\left(g\left(\bar{\xi}_{c}+z\right)\right),
$$

and we obtain

$$
\partial_{t} \vec{V}+\left(\vec{V} \cdot \nabla_{\vec{\xi}}\right) \vec{V}+A A^{t} \nabla_{\vec{\xi}}(P+g(\bar{\xi} c+z))=\vec{\Gamma}(\vec{V}),
$$

with

$$
\vec{\Gamma}(\vec{V})=\left(\left(\vec{V} \cdot \nabla_{\vec{\xi}}\right) A\right) A^{-1} \vec{V}=-A\left(\left(\vec{V} \cdot \nabla_{\vec{\xi}}\right) A^{-1}\right) \vec{V},
$$

the Christoffel symbol of the transformation. Denoting the components of (3.23) by $\vec{\Gamma} \equiv(\boldsymbol{\Gamma}, \bar{\Gamma}),(3.22)$ can also be written with $(3.6)$

$$
\begin{gathered}
\partial_{t} \mathbf{V}+\left(\vec{V} \cdot \nabla_{\vec{\xi}}\right) \mathbf{V}+M \nabla_{\boldsymbol{\xi}}(P+g(\bar{\xi} c+z))=\boldsymbol{\Gamma}(\vec{V}), \\
\partial_{t} \bar{V}+\left(\vec{V} \cdot \nabla_{\vec{\xi}}\right) \bar{V}+\partial_{\bar{\xi}} P+g c=\bar{\Gamma}(\vec{V}),
\end{gathered}
$$

with $M$ defined by (3.6). To simplify notation, we will write $\partial_{i}=\partial_{\vec{\xi}_{i}}$, for indices $i=1, \ldots, N+1$. We compute with (3.3)-(3.4)

$$
\begin{aligned}
& \left(\begin{array}{rr}
\partial_{\xi} \mathbf{X} & 0 \\
0 & 1
\end{array}\right) A\left(\partial_{i}\left(A^{-1}\right)\right) \\
& =\left(\begin{array}{cc}
\mathrm{Id}-\mathbf{s s}^{t} c \mathbf{s} \\
-\mathbf{s}^{t} & c
\end{array}\right)\left(\begin{array}{cc}
\partial_{i}\left(\partial_{\boldsymbol{\xi}} \mathbf{X}\right) & -\partial_{i} \mathbf{s} \\
\frac{1}{c} \mathbf{s}^{t}\left(\partial_{i}\left(\partial_{\boldsymbol{\xi}} \mathbf{X}\right)\right)+\left(\partial_{i}\left(\frac{1}{c} \mathbf{s}^{t}\right)\right)\left(\partial_{\boldsymbol{\xi}} \mathbf{X}\right) & \partial_{i} c
\end{array}\right)
\end{aligned}
$$

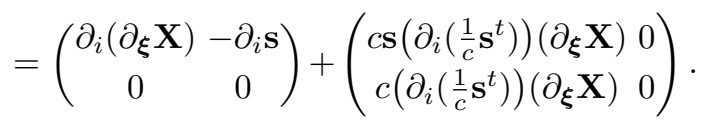

Let us consider the last component $\vec{\Gamma}(\vec{V})$ of $\vec{\Gamma}(\vec{V})$ first. From definition (2.1) above we know that $\frac{1}{c} \mathbf{s}^{t}=\partial_{x} z$. Hence the chain rule gives

$$
\bar{\Gamma}(\vec{V})=-c \mathbf{V}^{t}\left(\partial_{\boldsymbol{\xi}} \mathbf{x}\right)^{t}\left(\partial_{x x}^{2} z\right)\left(\partial_{\boldsymbol{\xi}} \mathbf{X}\right) \mathbf{V} .
$$

Next, we have

$$
\begin{aligned}
\left(\partial_{\boldsymbol{\xi}} \mathbf{X}\right) \mathbf{\Gamma}(\vec{V}) & =-\left(\left(\partial_{\vec{\xi}}\left(\partial_{\boldsymbol{\xi}} \mathbf{X}\right)\right) \vec{V}\right) \mathbf{V}+\left(\partial_{\boldsymbol{\xi}} \mathbf{s}\right) \mathbf{V} \bar{V}+\bar{\Gamma}(\vec{V}) \mathbf{s} \\
& =-\partial_{\boldsymbol{\xi} \boldsymbol{\xi}}^{2} \mathbf{X} \cdot \mathbf{V} \cdot \mathbf{V}+2\left(\partial_{\boldsymbol{\xi}} \mathbf{s}\right) \mathbf{V} \bar{V}+\bar{\Gamma}(\vec{V}) \mathbf{s} .
\end{aligned}
$$

The formulas (3.20) and (3.24)-(3.27) conclude the derivation of the transformed incompressible Euler equations inside the domain $\Omega_{t}$.

Concerning boundary conditions, (3.12) and (3.13) are unchanged since $\vec{U} \cdot \vec{n}=\bar{V}$, and we only need to give an equation for (3.14). Since $\nabla_{\vec{X}} \cdot \vec{U}=0$, this condition of advection of the domain can be written

$$
\partial_{t} \mathbf{I}_{\vec{X} \in \Omega_{t}}+\nabla_{\vec{X}} \cdot\left(\mathbf{I}_{\vec{X} \in \Omega_{t}} \vec{U}\right)=0 \quad \text { for } 0<\bar{\xi}<\infty,
$$


thus multiplying by $J$ and using the divergence chain rule, it becomes

$$
\partial_{t}\left(J \mathbf{I}_{\bar{\xi}<h(t, \boldsymbol{\xi})}\right)+\nabla_{\vec{\xi}} \cdot\left(J \mathbf{I}_{\bar{\xi}<h(t, \boldsymbol{\xi})} \vec{V}\right)=0 \quad \text { for } 0<\bar{\xi}<\infty .
$$

Since this equation is trivial inside the domain, only the part proportional to $\delta(h(t, \boldsymbol{\xi})-\bar{\xi})$ is involved, thus $(3.29)$ can be explicited as

$$
\partial_{t} h+\mathbf{V}_{\bar{\xi}=h(t, \boldsymbol{\xi})} \cdot \nabla_{\boldsymbol{\xi}} h=\bar{V}_{\bar{\xi}=h(t, \boldsymbol{\xi})} \cdot
$$

In order to put this equation for $h$ in conservation form, we use the assumption $\bar{V}=0$ at $\bar{\xi}=0$ in $(3.29)$, which gives

$$
\partial_{t}\left(J \mathbf{I}_{0<\bar{\xi}<h(t, \boldsymbol{\xi})}\right)+\nabla_{\vec{\xi}} \cdot\left(J \mathbf{I}_{0<\bar{\xi}<h(t, \boldsymbol{\xi})} \vec{V}\right)=0 \quad \text { for }-\infty<\bar{\xi}<\infty
$$

Integrating in $\bar{\xi}$ from $-\infty$ to $+\infty$ we obtain

$$
\partial_{t} \int_{0}^{h(t, \boldsymbol{\xi})} J d \bar{\xi}+\nabla_{\boldsymbol{\xi}} \cdot \int_{0}^{h(t, \boldsymbol{\xi})} J \mathbf{V} d \bar{\xi}=0 .
$$

This last formulation is the true conservative formulation, since the total volume of material is $\int_{\vec{X} \in \Omega_{t}} d \vec{X}=\iint_{0}^{h} J d \bar{\xi} d \boldsymbol{\xi}$.

3.2.2. Second velocity decomposition: the normal form. Our second choice of new velocity components is to define

$$
\vec{W} \equiv(\mathbf{W}, \bar{W})=A^{-t} \vec{U}
$$

The relation between $\vec{W}$ and $\vec{V}$ defined in (3.15) is then given by

$$
\vec{W}=A^{-t} A^{-1} \vec{V}
$$

thus according to (3.6),

$$
\mathbf{W}=M^{-1} \mathbf{V}, \quad \bar{W}=\bar{V} .
$$

With this choice, we have

$$
|\vec{U}|^{2}=\vec{U} \cdot A^{t} \vec{W}=\vec{W} \cdot \vec{V}=\vec{W}^{t} A A^{t} \vec{W}=\mathbf{W}^{t} M \mathbf{W}+\bar{W}^{2} .
$$

Now we multiply (3.10) by $A^{-t}$. From (3.16), (3.8) and (3.21), we get

$$
\partial_{t} \vec{W}+\left(\vec{V} \cdot \nabla_{\vec{\xi}}\right) \vec{W}+\nabla_{\vec{\xi}}(P+g(\bar{\xi} c+z))=\vec{\Xi},
$$

with

$$
\vec{\Xi} \equiv\left(\left(\vec{V} \cdot \nabla_{\vec{\xi}}\right) A^{-t}\right) \vec{U}=\left(\left(\vec{V} \cdot \nabla_{\vec{\xi}}\right) A^{-t}\right) A^{-1} \vec{V}
$$

Denoting as before $\partial_{i}=\partial_{\vec{\xi}_{i}}$, for indices $i=1, \ldots, N+1$, we have by definition $A_{k j}^{-t}=$ $\partial_{k} \vec{X}_{j}$, hence

$$
\partial_{i} A_{k l}^{-t}=\partial_{i k}^{2} \vec{X}_{l}=\partial_{k} A_{i l}^{-t}
$$


and therefore denoting

$$
\mathcal{G}=A^{-t} A^{-1}=\left(\begin{array}{cc}
M^{-1} & 0 \\
0 & 1
\end{array}\right)
$$

we compute

$$
\begin{aligned}
& \sum_{l}\left(\partial_{i} A_{k l}^{-t}\right) A_{l j}^{-1}=\sum_{l}\left(\partial_{k} A_{i l}^{-t}\right) A_{l j}^{-1} \\
& =\partial_{k} \mathcal{G}_{i j}-\sum_{l} A_{i l}^{-t}\left(\partial_{k} A_{l j}^{-1}\right) \\
& =\partial_{k} \mathcal{G}_{i j}-\sum_{l} A_{l i}^{-1}\left(\partial_{k} A_{j l}^{-t}\right)=\partial_{k} \mathcal{G}_{i j}-\sum_{l}\left(\partial_{j} A_{k l}^{-t}\right) A_{l i}^{-1} .
\end{aligned}
$$

When multiplying by $\vec{V}_{i} \vec{V}_{j}$ and summing over $i$ and $j$, the second term on the right gives the same as the sum on the left. Thus we get

$$
\begin{aligned}
\vec{\Xi}_{k} & =\frac{1}{2} \vec{V} \cdot\left(\partial_{k} \mathcal{G}\right) \vec{V} \\
& =\frac{1}{2} \vec{W} \cdot \mathcal{G}^{-1}\left(\partial_{k} \mathcal{G}\right) \mathcal{G}^{-1} \vec{W} \\
& =-\frac{1}{2} \vec{W} \cdot\left(\partial_{k} \mathcal{G}^{-1}\right) \vec{W}=-\frac{1}{2} \mathbf{W}^{t}\left(\partial_{k} M\right) \mathbf{W} .
\end{aligned}
$$

Since $\bar{W}=\bar{V}$, comparing (3.25) to the last component of (3.37), this gives a new formula instead of $(3.26)$,

$$
\bar{\Gamma}(\vec{V})=\bar{\Xi}=-\frac{1}{2} \mathbf{W}^{t}\left(\partial_{\bar{\xi}} M\right) \mathbf{W} .
$$

Using now (3.41), (3.42) in (3.37), we obtain

$$
\begin{aligned}
& \partial_{t} \mathbf{W}+\left(\vec{V} \cdot \nabla_{\vec{\xi}}\right) \mathbf{W}+\nabla_{\boldsymbol{\xi}}\left(\frac{1}{2} \mathbf{W}^{t} M \mathbf{W}+P+g(\bar{\xi} c+z)\right)=\left(\partial_{\boldsymbol{\xi}} \mathbf{W}\right)^{t} M \mathbf{W}, \\
& \partial_{t} \bar{W}+\left(\vec{V} \cdot \nabla_{\vec{\xi}}\right) \bar{W}+\partial_{\bar{\xi}}\left(\frac{1}{2} \mathbf{W}^{t} M \mathbf{W}+P+g(\bar{\xi} c+z)\right)=\left(\partial_{\bar{\xi}} \mathbf{W}\right)^{t} M \mathbf{W} .
\end{aligned}
$$

Notice that in (3.43), we can make the curl of $\mathbf{W}$ appear by writing

$$
\left(\vec{V} \cdot \nabla_{\vec{\xi}}\right) \mathbf{W}-\left(\partial_{\boldsymbol{\xi}} \mathbf{W}\right)^{t} M \mathbf{W}=\bar{V} \partial_{\bar{\xi}} \mathbf{W}+\left(\partial_{\boldsymbol{\xi}} \mathbf{W}-\left(\partial_{\boldsymbol{\xi}} \mathbf{W}\right)^{t}\right) M \mathbf{W}
$$

These two equations (3.43), (3.44) conclude the derivation of the transformed Euler equations for our second velocity decomposition, since the divergence equation (3.20) and the boundary conditions (3.12) (note that $\bar{V}=\bar{W}=\vec{n} \cdot \vec{U}),(3.13)$, and (3.30) or (3.32) remain unchanged, we just have to say that $\mathbf{V}=M \mathbf{W}$.

3.3. Shallow water approximation for small slope variation. We derive in this section the model of Subsection 2.2, i.e., we prove Theorem 2.1 (iii). This is done starting from the formulation of incompressible Euler equations of Subsection 3.2.1. In order to emphasize the question of velocity variation in the layer, we first perform the derivation within the classical assumption of almost constant dependency, and treat general dependency afterwards. 
3.3.1. Almost constant velocity in the layer. We assume that

(a) the material layer is thin, $h=\mathcal{O}(\epsilon)$,

(b) the curvature is small, $\mathcal{H}=\mathcal{O}(\epsilon)$,

(c) the velocity $\mathbf{V}$ does almost not depend on the normal variable $\bar{\xi}$,

$$
\mathbf{V}(t, \vec{\xi})=V(t, \boldsymbol{\xi})+\mathcal{O}\left(\epsilon^{2}\right)
$$

Here, $\epsilon \ll 1$ is the aspect ratio between the width of the layer and the typical length of phenomena in $x$. Note that (b) can also be written as $\partial_{x} \mathbf{s}=\mathcal{O}(\epsilon)$. Assumption (c) is made to get a model in the variable $\boldsymbol{\xi}$ only. The usual procedure to get an equation in $\boldsymbol{\xi}$ is depth-integration, but here we shall write the equation in the full domain $\Omega_{t}$, and check that the variable $\bar{\xi}$ disappears. This approach has the advantage of justifying the compatibility of assumption (c) with the set of equations in the whole material layer.

With (a) and (3.2), we get $\bar{\xi}=\mathcal{O}(\epsilon)$. Assuming moreover that there is no boundary layer, we deduce from the boundary condition (3.12) at the bed that

$$
\bar{V}=\vec{n} \cdot \vec{U}=\mathcal{O}(\epsilon)
$$

We can now make expansions in (3.5), (3.26) and (3.27),

$$
\begin{gathered}
\partial_{\boldsymbol{\xi}} \mathbf{X}=\partial_{\boldsymbol{\xi}} \mathbf{x}+\mathcal{O}\left(\epsilon^{2}\right) \\
\bar{\Gamma}(\vec{V})=-c V^{t}\left(\partial_{\boldsymbol{\xi}} \mathbf{x}\right)^{t}\left(\partial_{x x}^{2} z\right)\left(\partial_{\boldsymbol{\xi}} \mathbf{x}\right) V+\mathcal{O}\left(\epsilon^{3}\right)=\mathcal{O}(\epsilon) \\
\left(\partial_{\boldsymbol{\xi}} \mathbf{x}\right) \mathbf{\Gamma}(\vec{V})=-\partial_{\boldsymbol{\xi} \boldsymbol{}}^{2} \mathbf{x} \cdot V \cdot V+\bar{\Gamma}(\vec{V}) \mathbf{s}+\mathcal{O}\left(\epsilon^{2}\right)
\end{gathered}
$$

Using this in (3.24), (3.25), we obtain the reduced momentum equation

$$
\begin{gathered}
\partial_{t} V+V \cdot \nabla_{\boldsymbol{\xi}} V+M \nabla_{\boldsymbol{\xi}}(P+g(\bar{\xi} c+z)) \\
=-\left(\partial_{\boldsymbol{\xi}} \mathbf{x}\right)^{-1} \partial_{\boldsymbol{\xi} \boldsymbol{}}^{2} \mathbf{x} \cdot V \cdot V+\bar{\Gamma}(\vec{V})\left(\partial_{\boldsymbol{\xi}} \mathbf{x}\right)^{-1} \mathbf{s}+\mathcal{O}\left(\epsilon^{2}\right),
\end{gathered}
$$

and the pressure relation

$$
\partial_{\bar{\xi}} P=-g c+\mathcal{O}(\epsilon)
$$

This equation can be integrated with the boundary condition (3.13),

$$
P=-g c(\bar{\xi}-h(t, \boldsymbol{\xi}))+\mathcal{O}\left(\epsilon^{2}\right) .
$$

Using this in (3.51) and expanding $M$ with (3.6), (3.48), we get our equation (2.10), up to terms in $\mathcal{O}\left(\epsilon^{2}\right)$. Finally, we have to write the equation for evolving the free surface. We use the formulation (3.32), and expanding $J$ according to (3.7), (3.48), we get $J=J_{0}+\mathcal{O}\left(\epsilon^{2}\right)$, and

$$
\partial_{t}\left(J_{0} h\right)+\nabla_{\boldsymbol{\xi}} \cdot\left(J_{0} h V\right)=\mathcal{O}\left(\epsilon^{3}\right),
$$

which is (2.9) up to terms in $\mathcal{O}\left(\epsilon^{3}\right)$. 
3.3.2. Arbitrary velocity dependency in the layer. We now establish Theorem 2.1 (iii) in its full generality, i.e. with the only assumptions

(a) the material layer is thin, $h=\mathcal{O}(\epsilon)$,

(b) the curvature is small, $\mathcal{H}=\mathcal{O}(\epsilon)$.

Now, the assumption (c) above is replaced by a linear approximation argument, and by an interpretation of (2.10) as the equation on the mean value of the velocity in the layer.

We first notice that since $\bar{\xi}=\mathcal{O}(\epsilon)$ by (a) and (3.2), the fact that we do not consider any singular boundary layer implies that up to an error in $\epsilon^{2}$, the normal dependency is linear,

$$
\mathbf{V}(t, \vec{\xi})=V(t, \boldsymbol{\xi})+V^{1}(t, \boldsymbol{\xi})(\bar{\xi}-h(t, \boldsymbol{\xi}) / 2)+\mathcal{O}\left(\epsilon^{2}\right),
$$

for some functions $V(t, \boldsymbol{\xi})$ and $V^{1}(t, \boldsymbol{\xi})$ that represent respectively the mean value and the slope of the velocity in the material layer. Then, the important point in evaluating integrals over the layer is that for any smooth function $\varphi$,

$$
\int_{0}^{h(t, \boldsymbol{\xi})} \varphi(\mathbf{V}(t, \vec{\xi})) d \bar{\xi}=h(t, \boldsymbol{\xi}) \varphi(V(t, \boldsymbol{\xi}))+\mathcal{O}\left(\epsilon^{3}\right) .
$$

We now follow the lines of the previous subsection and observe that from (3.12), (3.5), (3.6), (3.7), (3.26), (3.27),

$$
\begin{gathered}
\bar{V}=\mathcal{O}(\epsilon) \\
\partial_{\boldsymbol{\xi}} \mathbf{X}=\partial_{\boldsymbol{\xi}} \mathbf{x}+\mathcal{O}\left(\epsilon^{2}\right), \\
M=\left(\partial_{\boldsymbol{\xi}} \mathbf{x}\right)^{-1}\left(\mathrm{Id}-\mathbf{s s}^{t}\right)\left(\partial_{\boldsymbol{\xi}} \mathbf{x}\right)^{-t}+\mathcal{O}\left(\epsilon^{2}\right), \\
J=J_{0}+\mathcal{O}\left(\epsilon^{2}\right), \\
\bar{\Gamma}(\vec{V})=-c V^{t}\left(\partial_{\boldsymbol{\xi}} \mathbf{x}\right)^{t}\left(\partial_{x x}^{2} z\right)\left(\partial_{\boldsymbol{\xi}} \mathbf{x}\right) V+\mathcal{O}\left(\epsilon^{2}\right)=\mathcal{O}(\epsilon), \\
\left(\partial_{\boldsymbol{\xi}} \mathbf{x}\right) \mathbf{\Gamma}(\vec{V})=-\partial_{\xi \xi}^{2} \mathbf{x} \cdot \mathbf{V} \cdot \mathbf{V}+\bar{\Gamma}(\vec{V}) \mathbf{s}+\mathcal{O}\left(\epsilon^{2}\right) .
\end{gathered}
$$

Using this in (3.25) we get with (3.13)

$$
\begin{gathered}
\partial_{\bar{\xi}} P=-g c+\mathcal{O}(\epsilon), \quad P=-g c(\bar{\xi}-h(t, \boldsymbol{\xi}))+\mathcal{O}\left(\epsilon^{2}\right), \\
P+g(\bar{\xi} c+z)=g(h c+z)+\mathcal{O}\left(\epsilon^{2}\right) .
\end{gathered}
$$

Concerning the continuity equation, we deduce by expanding (3.32) with (3.56) that (2.9) holds, up to terms in $\mathcal{O}\left(\epsilon^{3}\right)$.

Now it remains only to deal with the velocity equation (3.24). If in this equation we replace $\mathbf{V}$ by its expansion (3.55), drop terms in $\epsilon^{2}$ and separate the terms constant and linear in $\bar{\xi}$, we obtain two equations coupling $V$ and $V^{1}$, one starting with $\partial_{t} V$ and another starting with $\partial_{t} V^{1}$. The striking property that we are going to justify now is that indeed the equation on $V$ is exactly (2.10), and in particular it is decoupled 
from $V^{1}$. The argument is as follows. Since in (3.55) $V$ is defined only up to terms in $\epsilon^{2}$, we can fix its value to the value of $\mathbf{V}$ at $\bar{\xi}=h(t, \boldsymbol{\xi}) / 2$, i.e., as

$$
V(t, \boldsymbol{\xi})=\mathbf{V}(t, \boldsymbol{\xi}, h(t, \boldsymbol{\xi}) / 2) .
$$

Then by the chain rule,

$$
\begin{aligned}
\left(\partial_{t} \mathbf{V}\right)(t, \boldsymbol{\xi}, h(t, \boldsymbol{\xi}) / 2) & =\partial_{t} V-\left(\partial_{\bar{\xi}} \mathbf{V}\right)(t, \boldsymbol{\xi}, h(t, \boldsymbol{\xi}) / 2) \partial_{t} h / 2 \\
\left(\partial_{\boldsymbol{\xi}} \mathbf{V}\right)(t, \boldsymbol{\xi}, h(t, \boldsymbol{\xi}) / 2) & =\partial_{\boldsymbol{\xi}} V-\left(\partial_{\bar{\xi}} \mathbf{V}\right)(t, \boldsymbol{\xi}, h(t, \boldsymbol{\xi}) / 2) \partial_{\boldsymbol{\xi}} h / 2
\end{aligned}
$$

We deduce the value of

$$
\partial_{t} \mathbf{V}+\left(\vec{V} \cdot \nabla_{\vec{\xi}}\right) \mathbf{V}=\partial_{t} \mathbf{V}+\left(\mathbf{V} \cdot \nabla_{\boldsymbol{\xi}}\right) \mathbf{V}+\bar{V} \partial_{\bar{\xi}} \mathbf{V}
$$

at the point $(t, \boldsymbol{\xi}, h(t, \boldsymbol{\xi}) / 2)$,

$$
\begin{aligned}
& \left(\partial_{t} \mathbf{V}+\left(\vec{V} \cdot \nabla_{\vec{\xi}}\right) \mathbf{V}\right)(t, \boldsymbol{\xi}, h(t, \boldsymbol{\xi}) / 2) \\
& =\partial_{t} V+\left(V \cdot \nabla_{\boldsymbol{\xi}}\right) V \\
& +\left(\partial_{\bar{\xi}} \mathbf{V}\right)(t, \boldsymbol{\xi}, h(t, \boldsymbol{\xi}) / 2)\left\{\bar{V}(t, \boldsymbol{\xi}, h(t, \boldsymbol{\xi}) / 2)-\partial_{t} h / 2-V \cdot \nabla_{\boldsymbol{\xi}} h / 2\right\} .
\end{aligned}
$$

But using condition (3.30) and since $\mathbf{V}(\bar{\xi}=h)=V+\mathcal{O}(\epsilon)$, we have

$$
\begin{aligned}
\bar{V}(t, \boldsymbol{\xi}, h(t, \boldsymbol{\xi}) / 2) & =\frac{1}{2} \bar{V}(t, \boldsymbol{\xi}, h(t, \boldsymbol{\xi}))+\mathcal{O}\left(\epsilon^{2}\right) \\
& =\left(\partial_{t} h / 2+V \cdot \nabla_{\boldsymbol{\xi}} h / 2\right)+\mathcal{O}\left(\epsilon^{2}\right) .
\end{aligned}
$$

Putting this in (3.68) we obtain

$$
\left(\partial_{t} \mathbf{V}+\left(\vec{V} \cdot \nabla_{\vec{\xi}}\right) \mathbf{V}\right)(t, \boldsymbol{\xi}, h(t, \boldsymbol{\xi}) / 2)=\partial_{t} V+\left(V \cdot \nabla_{\boldsymbol{\xi}}\right) V+\mathcal{O}\left(\epsilon^{2}\right),
$$

and $V^{1}=\partial_{\bar{\xi}} \mathbf{V}+\mathcal{O}(\epsilon)$ disappears. Now in order to obtain our velocity equation, we take the value of $(3.24)$ at $\bar{\xi}=h(t, \boldsymbol{\xi}) / 2$. Using (3.70), we obtain (2.10) up to terms in $\mathcal{O}\left(\epsilon^{2}\right)$.

Finally, the justification of the relation with the physical velocity $(2.8)$ is that in our expansion $\bar{V}=\vec{n} \cdot \vec{U}=\mathcal{O}(\epsilon)$, thus from (3.19)

$$
\vec{U}=\mathcal{V}^{t g}+\mathcal{O}(\epsilon)
$$

A higher order expansion is also possible,

$$
\mathcal{V}^{t g}=(\vec{U}-(\vec{n} \cdot \vec{U}) \vec{n})(t, \boldsymbol{\xi}, h(t, \boldsymbol{\xi}) / 2)+\mathcal{O}\left(\epsilon^{2}\right) .
$$

Remark. Note that assumption (c) in Subsection 3.3.1 (see (3.46)) is consistent with the derivation we gave here since it is always possible to take $V^{1}=0$ in (3.55). Indeed the equation on $V^{1}$, that we did not write down explicitly but that can be obtained as explained above, is linear (with coefficients depending on $V$ ). Thus if we start from initial data with $V^{1}$ vanishing, then $V^{1}$ vanishes for all time. 
3.4. Shallow water approximation for arbitrary topography. We derive in this section the model of Subsection 2.3, i.e., we prove Theorem 2.3 (iii). This is done starting from the formulation of incompressible Euler equations of Subsection 3.2.2. The only assumption is that the material layer is thin, $h=\mathcal{O}(\epsilon)$. Contrary to the case of small curvature, we cannot assume here that the velocity is almost constant in the layer, as this would lead to a contradiction unless $\operatorname{curl}_{\boldsymbol{\xi}} W=\mathcal{O}(\epsilon)$. Therefore, we rather follow the strategy of Subsection 3.3.2.

We still have that $\bar{\xi}=\mathcal{O}(\epsilon)$, and since we do not consider any singular boundary layer, the normal dependency of the velocity is linear up to an error in $\epsilon^{2}$,

$$
\mathbf{W}(t, \vec{\xi})=W(t, \boldsymbol{\xi})+W^{1}(t, \boldsymbol{\xi})(\bar{\xi}-h(t, \boldsymbol{\xi}) / 2)+\mathcal{O}\left(\epsilon^{2}\right),
$$

for some mean value $W(t, \boldsymbol{\xi})$ and some slope $W^{1}(t, \boldsymbol{\xi})$. Then, by the mean value formula (3.56) and since $\mathbf{V}=M \mathbf{W}$, the free surface evolution equation (3.32) directly gives (2.35) up to an error in $\mathcal{O}\left(\epsilon^{3}\right)$. Next, since $\bar{W}=\bar{V}=\vec{n} \cdot \vec{U}=\mathcal{O}(\epsilon)$ by (3.12), equation (3.44) gives

$$
\partial_{\bar{\xi}}\left(\frac{1}{2} \mathbf{W}^{t} M \mathbf{W}+P+g(\bar{\xi} c+z)\right)=\left(\partial_{\bar{\xi}} \mathbf{W}\right)^{t} M \mathbf{W}+\mathcal{O}(\epsilon) .
$$

Therefore, by integration between $\bar{\xi}$ and $h(t, \boldsymbol{\xi})$ and using (3.13),

$$
\begin{aligned}
& \frac{1}{2} \mathbf{W}^{t} M \mathbf{W}+P+g(\bar{\xi} c+z) \\
& =\frac{1}{2} \mathbf{W}_{h}^{t} M_{h} \mathbf{W}_{h}+g(h c+z)-\int_{\bar{\xi}}^{h} \partial_{\bar{\xi}} \mathbf{W}^{t} M \mathbf{W}+\mathcal{O}\left(\epsilon^{2}\right) \\
& =\frac{1}{2} \mathbf{W}^{t} M_{h} \mathbf{W}+g(h c+z)-\int_{\bar{\xi}}^{h} \partial_{\bar{\xi}} \mathbf{W}^{t}\left(M-M_{h}\right) \mathbf{W}+\mathcal{O}\left(\epsilon^{2}\right) \\
& =\frac{1}{2} \mathbf{W}^{t} M_{h} \mathbf{W}+g(h c+z)+\mathcal{O}\left(\epsilon^{2}\right),
\end{aligned}
$$

where the index $h$ means that we take the value at $\bar{\xi}=h(t, \boldsymbol{\xi})$. Now, we can replace in (3.43) $\mathbf{W}$ by its expansion (3.73) and the term under $\nabla_{\boldsymbol{\xi}}$ by its expansion in (3.75). Separating the terms constant and linear in $\bar{\xi}$, the result is a system of two equations involving $W$ and $W^{1}$. We claim that again, the equation on $W$ is decoupled from $W^{1}$. In order to obtain this equation, we argue as in Subsection 3.3.2. Since in (3.73) $W$ is only defined up to terms in $\epsilon^{2}$, we can fix its value to the value of $\mathbf{W}$ at $\bar{\xi}=h(t, \boldsymbol{\xi}) / 2$,

$$
W(t, \boldsymbol{\xi})=\mathbf{W}(t, \boldsymbol{\xi}, h(t, \boldsymbol{\xi}) / 2) .
$$

Then the same computations (3.66)-(3.69) give

$$
\begin{aligned}
\left(\partial_{t} \mathbf{W}\right)(t, \boldsymbol{\xi}, h(t, \boldsymbol{\xi}) / 2) & =\partial_{t} W-\left(\partial_{\bar{\xi}} \mathbf{W}\right)(t, \boldsymbol{\xi}, h(t, \boldsymbol{\xi}) / 2) \partial_{t} h / 2, \\
\left(\partial_{\boldsymbol{\xi}} \mathbf{W}\right)(t, \boldsymbol{\xi}, h(t, \boldsymbol{\xi}) / 2) & =\partial_{\boldsymbol{\xi}} W-\left(\partial_{\bar{\xi}} \mathbf{W}\right)(t, \boldsymbol{\xi}, h(t, \boldsymbol{\xi}) / 2) \partial_{\boldsymbol{\xi}} h / 2,
\end{aligned}
$$

and

$$
\begin{aligned}
& \left(\partial_{t} \mathbf{W}+\left(\vec{V} \cdot \nabla_{\vec{\xi}}\right) \mathbf{W}\right)(t, \boldsymbol{\xi}, h(t, \boldsymbol{\xi}) / 2) \\
& =\partial_{t} W+\left(M_{h / 2} W\right) \cdot \nabla_{\boldsymbol{\xi}} W \\
& +\left(\partial_{\bar{\xi}} \mathbf{W}\right)(t, \boldsymbol{\xi}, h(t, \boldsymbol{\xi}) / 2) \\
& \quad \times\left\{\bar{V}(t, \boldsymbol{\xi}, h(t, \boldsymbol{\xi}) / 2)-\partial_{t} h / 2-\left(M_{h / 2} W\right) \cdot \nabla_{\boldsymbol{\xi}} h / 2\right\} \\
& =\partial_{t} W+\left(M_{h / 2} W\right) \cdot \nabla_{\boldsymbol{\xi}} W+\mathcal{O}\left(\epsilon^{2}\right) .
\end{aligned}
$$


Now, we take the value of $(3.43)$ at $\bar{\xi}=h(t, \boldsymbol{\xi}) / 2$. We apply (3.78) and (3.75), and we use again the chain rule formula (3.77) for the two terms involving derivatives in $\boldsymbol{\xi}$. For each term it produces an extra term proportional to $\partial_{\boldsymbol{\xi}} h$, thus in $\mathcal{O}(\epsilon)$. But since $M_{h}-M_{h / 2}=\mathcal{O}(\epsilon)$, the errors compensate and we obtain

$$
\begin{aligned}
& \partial_{t} W+\left(M_{h / 2} W\right) \cdot \nabla_{\boldsymbol{\xi}} W+\nabla_{\boldsymbol{\xi}}\left(\frac{1}{2} W^{t} M_{h} W+g(h c+z)\right) \\
= & \left(\partial_{\boldsymbol{\xi}} W\right)^{t} M_{h / 2} W+\mathcal{O}\left(\epsilon^{2}\right),
\end{aligned}
$$

which is (2.36) up to terms in $\mathcal{O}\left(\epsilon^{2}\right)$. Concerning the formula (2.34) for $\mathcal{V}^{t g}$, it follows from (3.19) and $\mathbf{V}=M \mathbf{W}$, which give

$$
\vec{U}-(\vec{n} \cdot \vec{U}) \vec{n}=\left(\begin{array}{c}
\mathrm{Id} \\
\frac{1}{c} \mathbf{s}^{t}
\end{array}\right)\left(\partial_{\boldsymbol{\xi}} \mathbf{X}\right) M \mathbf{W} .
$$

Since by (3.6) we have $\left(\partial_{\boldsymbol{\xi}} \mathbf{X}\right) M=\left(\mathrm{Id}-\mathbf{s s}^{t}\right)\left(\partial_{\boldsymbol{\xi}} \mathbf{X}\right)^{-t}$, with (3.5) we get

$$
(\vec{U}-(\vec{n} \cdot \vec{U}) \vec{n})(t, \boldsymbol{\xi}, h(t, \boldsymbol{\xi}) / 2)=\mathcal{V}^{t g} .
$$

To conclude this section, we would like to mention that the conservative momentum and energy equations of our models can also be obtained by expansions from corresponding equations for the incompressible equations in the layer. To obtain the energy equation (2.43) for example, we multiply (3.10) by $\vec{U}$ to get

$$
\partial_{t}|\vec{U}|^{2} / 2+\left(\vec{U} \cdot \nabla_{\vec{X}}\right)|\vec{U}|^{2} / 2+\vec{U} \cdot \nabla_{\vec{X}} P=-\vec{U} \cdot \vec{g}=-\vec{U} \cdot \nabla_{\vec{X}}(\vec{g} \cdot \vec{X}),
$$

which can be put in conservative form with (3.11),

$$
\partial_{t}|\vec{U}|^{2} / 2+\nabla_{\vec{X}} \cdot\left(\left(|\vec{U}|^{2} / 2+P+\vec{g} \cdot \vec{X}\right) \vec{U}\right)=0 .
$$

Thus multiplying by $J$ and using the divergence chain rule,

$$
\partial_{t}\left(J|\vec{U}|^{2} / 2\right)+\nabla_{\vec{\xi}} \cdot\left(J\left(|\vec{U}|^{2} / 2+P+\vec{g} \cdot \vec{X}\right) A \vec{U}\right)=0 .
$$

Then, using (3.36) and (3.1),

$$
\begin{gathered}
\partial_{t}\left\{J\left(\frac{1}{2}\left(\mathbf{W}^{t} M \mathbf{W}+\bar{W}^{2}\right)+g(\bar{\xi} c+z)\right)\right\} \\
+\nabla_{\vec{\xi}} \cdot\left\{J\left(\frac{1}{2}\left(\mathbf{W}^{t} M \mathbf{W}+\bar{W}^{2}\right)+P+g(\bar{\xi} c+z)\right) \vec{V}\right\}=0 .
\end{gathered}
$$

Integrating for $\bar{\xi}$ between 0 and $h$ and using the boundary condition (3.30) we obtain

$$
\begin{aligned}
& \partial_{t} \int_{0}^{h} J\left(\frac{1}{2}\left(\mathbf{W}^{t} M \mathbf{W}+\bar{W}^{2}\right)+g(\bar{\xi} c+z)\right) d \bar{\xi} \\
& +\nabla_{\boldsymbol{\xi}} \cdot \int_{0}^{h} J\left(\frac{1}{2}\left(\mathbf{W}^{t} M \mathbf{W}+\bar{W}^{2}\right)+P+g(\bar{\xi} c+z)\right) M \mathbf{W} d \bar{\xi}=0 .
\end{aligned}
$$

Expanding the integrals we finally get (2.43) up to terms in $\mathcal{O}\left(\epsilon^{3}\right)$. A similar computation, expanding (3.100) (with $\nu=0$ ) with the help of (3.75) yields the momentum equation (2.41) up to terms in $\mathcal{O}\left(\epsilon^{3}\right)$. 
Remark. The proof above shows that if we have a solution to $(2.35)-(2.36)$ such that $\operatorname{curl}_{\boldsymbol{\xi}} W=0$, then $\mathbf{W} \equiv W$ is an exact solution constant in the variable $\bar{\xi}$ to the hydrostatic incompressible Euler system (where only the normal acceleration $\partial_{t} \bar{W}+\left(\vec{V} \cdot \nabla_{\vec{\xi}}\right) \bar{W}$ in (3.44) is removed).

3.5. Bottom Coulomb friction. In this section, we prove Theorems 2.4 and 2.5. Since Theorem 2.4 can take the equivalent form $(2.56)-(2.57)$, we are going to provide a common proof for both cases. We will derive the momentum equation in conservative form, i.e., we derive an equation analogous to (2.41) with friction terms added. Transforming this into the nonconservative form of (2.59) is then straightforward.

We start from the free boundary incompressible Navier-Stokes system in cartesian coordinates. We assume that the functions

$$
\vec{U}(t, \vec{X}) \in \mathbb{R}^{N+1}, \quad P(t, \vec{X}) \in \mathbb{R}
$$

for $t>0$ and $\vec{X} \in \Omega_{t}$ satisfy

$$
\begin{aligned}
\partial_{t} \vec{U}+\left(\vec{U} \cdot \nabla_{\vec{X}}\right) \vec{U}+\nabla_{\vec{X}} P & =-\vec{g}+\nabla_{\vec{X}} \cdot \sigma, \\
\nabla_{\vec{X}} \cdot \vec{U} & =0 .
\end{aligned}
$$

Again, $\vec{g}=g\left(\begin{array}{l}0 \\ 1\end{array}\right)$ with $g$ the gravitational constant, and

$$
\sigma=\nu\left(\partial_{\vec{X}} \vec{U}+\left(\partial_{\vec{X}} \vec{U}\right)^{t}\right)
$$

with suitable viscosity coefficient $\nu$. This problem is well-defined under the following set of boundary conditions which we will assume throughout. Let $\vec{N}$ be the outward unit normal vector at the free boundary of $\Omega_{t}$, see (3.112) below, and define the total stress tensor

$$
\sigma_{T}=P \operatorname{Id}-\sigma
$$

Then we require

- at the bed

$$
\left.\begin{array}{rl}
\vec{U} \cdot \vec{n} & =0 \\
\sigma_{T} \vec{n}-\left(\vec{n} \cdot \sigma_{T} \vec{n}\right) \vec{n} & =-\mu \frac{\vec{U}}{|\vec{U}|}\left(\vec{n} \cdot \sigma_{T} \vec{n}\right)_{+}
\end{array}\right\} \quad \text { for } \bar{\xi}=0,
$$

- at the free boundary

$$
\left.\begin{array}{r}
\begin{array}{r}
P \\
\sigma_{T} \vec{N}-\left(\vec{N} \cdot \sigma_{T} \vec{N}\right) \vec{N}
\end{array}=0
\end{array}\right\} \quad \text { for } \bar{\xi}=h(t, \boldsymbol{\xi}) .
$$

The first equation in (3.92) is the no-penetration condition which we already considered in Section 3.2, the second is the usual Coulomb friction condition. It states that the tangential part of the total stress tensor is opposite to the material velocity $\vec{U}$, and its ratio to the normal stress is given by $\mu$. Here again the positive part is used to neutralize the friction in case the material is leaving the bed, and $\vec{U} /|\vec{U}|$ should be understood as multivalued when $\vec{U}=0$. Condition (3.93) states that the atmospheric pressure and the tangential part of the total stress vector vanish at the free surface. 
Note that we can replace $\sigma_{T}$ by $-\sigma$ on the left-hand sides of (3.92) and (3.93) since the unit length of $\vec{n}$ and $\vec{N}$ implies that the terms with PId cancel.

We are going to transform equations (3.88)-(3.89), (3.92)-(3.93) into the curvilinear coordinate system $\vec{\xi}$ defined in (3.1), with the velocity decomposition of Subsection 3.2 .2 , i.e., with

$$
\vec{W}=A^{-t} \vec{U}, \quad A^{-1}=\partial_{\vec{\xi}} \vec{X} .
$$

We will need the following lemma on the divergence of tensors whose proof is given at the end of this section. For ease of notation we write $B_{1}: B_{2} \equiv \operatorname{tr}\left(B_{1} B_{2}\right)$ for matrices $B_{1}$ and $B_{2}$, with tr the trace.

Lemma 3.1. Let $\vec{\xi} \mapsto \vec{X}(\vec{\xi})$ be a change of coordinates, and let $\sigma$ be a symmetric tensor, $\sigma^{t}=\sigma$. Then

$$
J A^{-t} \nabla_{\vec{X}} \cdot \sigma=\nabla_{\vec{\xi}} \cdot\left(J \mathcal{P} A A^{t}\right)+\frac{J}{2} \mathcal{P}: \nabla_{\vec{\xi}}\left(A A^{t}\right)
$$

with $\mathcal{P}=A^{-t} \sigma A^{-1}, A^{-1}=\partial_{\vec{\xi}} \vec{X}$ and $J=\operatorname{det} A^{-1}$.

Note that $A A^{t}$ has block structure, see (3.6). In particular, the only nontrivial entry of any derivative of $A A^{t}$ is the left upper block. We will use Lemma 3.1 to transform the divergence of the tensor $\sigma$ into the curvilinear coordinate system. In order to simplify a bit the notation, we decompose the tensor $\mathcal{P}=A^{-t} \sigma A^{-1}$ into blocks as

$$
\mathcal{P}=\left(\begin{array}{cc}
\mathbf{P} & \mathbf{Z} \\
\mathbf{Z}^{t} & f
\end{array}\right) \quad \text { with } \quad \mathbf{P} \in \mathbb{R}^{N \times N}, \quad \mathbf{Z}, \bar{Z} \in \mathbb{R}^{N}, \quad f \in \mathbb{R} .
$$

We multiply (3.88) by $A^{-t}$. Following the arguments of Subsection 3.2.2 and using Lemma 3.1 yields the transformed momentum equation, separated in tangential and normal components as

$$
\begin{gathered}
\partial_{t} \mathbf{W}+\left(\vec{V} \cdot \nabla_{\vec{\xi}}\right) \mathbf{W}+\nabla_{\boldsymbol{\xi}}\left(\frac{1}{2} \mathbf{W}^{t} M \mathbf{W}+P+g(\bar{\xi} c+z)\right) \\
=\left(\partial_{\boldsymbol{\xi}} \mathbf{W}\right)^{t} M \mathbf{W}+J^{-1} \nabla_{\boldsymbol{\xi}} \cdot(J \mathbf{P} M)+J^{-1} \partial_{\bar{\xi}}(J \mathbf{Z})+\frac{1}{2} \mathbf{P}: \nabla_{\boldsymbol{\xi}} M, \\
\partial_{t} \bar{W}+\left(\vec{V} \cdot \nabla_{\vec{\xi}}\right) \bar{W}+\partial_{\bar{\xi}}\left(\frac{1}{2} \mathbf{W}^{t} M \mathbf{W}+P+g(\bar{\xi} c+z)\right) \\
=\left(\partial_{\bar{\xi}} \mathbf{W}\right)^{t} M \mathbf{W}+J^{-1} \nabla_{\boldsymbol{\xi}} \cdot(J M \mathbf{Z})+J^{-1} \partial_{\bar{\xi}}(J f)+\frac{1}{2} \mathbf{P}: \partial_{\bar{\xi}} M,
\end{gathered}
$$

see (3.43)-(3.44). Multiplying (3.97) by $J$ and using again the incompressibility condition (3.20) we deduce the conservative formulation

$$
\begin{aligned}
\partial_{t}(J \mathbf{W})+\nabla_{\vec{\xi}} \cdot(\mathbf{W} \otimes J \vec{V})+\nabla_{\boldsymbol{\xi}}(J(P+g(\bar{\xi} c+z))) \\
=-J \frac{1}{2} \mathbf{W}^{t}\left(\nabla_{\boldsymbol{\xi}} M\right) \mathbf{W}+(P+g(\bar{\xi} c+z)) \nabla_{\boldsymbol{\xi}} J \\
\quad+\nabla_{\boldsymbol{\xi}} \cdot(J \mathbf{P} M)+\partial_{\bar{\xi}}(J \mathbf{Z})+\frac{1}{2} J \mathbf{P}: \nabla_{\boldsymbol{\xi}} M .
\end{aligned}
$$

We integrate this equation for $\bar{\xi}$ between 0 and $h(t, \boldsymbol{\xi})$. This yields using the boundary 
conditions

$$
\begin{aligned}
& \partial_{t} \int_{0}^{h} J \mathbf{W} d \bar{\xi}+\nabla_{\boldsymbol{\xi}} \cdot \int_{0}^{h}(\mathbf{W} \otimes J \mathbf{V}) d \bar{\xi} \\
& \quad+\nabla_{\boldsymbol{\xi}} \int_{0}^{h}(J(P+g(\bar{\xi} c+z))) d \bar{\xi}-J_{h} g(h c+z) \nabla_{\boldsymbol{\xi}} h \\
& =\int_{0}^{h}\left(-J \frac{1}{2} \mathbf{W}^{t}\left(\partial_{\boldsymbol{\xi}_{i}} M\right) \mathbf{W}+(P+g(\bar{\xi} c+z)) \nabla_{\boldsymbol{\xi}} J\right) d \bar{\xi} \\
& \quad+\nabla_{\boldsymbol{\xi}} \cdot \int_{0}^{h} J \mathbf{P} M d \bar{\xi}+\frac{1}{2} \int_{0}^{h} J \mathbf{P}: \nabla_{\boldsymbol{\xi}} M d \bar{\xi} \\
& \quad+J_{h}\left(-\mathbf{P}_{h} M_{h} \nabla_{\boldsymbol{\xi}} h+\mathbf{Z}_{h}\right)-J_{0} \mathbf{Z}_{0},
\end{aligned}
$$

where the subscripts 0 and $h$ indicate that the terms are evaluated at the bottom and the free surface respectively. We used the Leibniz rule several times to commute integrals in $\bar{\xi}$ and derivatives in $\boldsymbol{\xi}$.

Now we will consider the stress tensor in more detail. Although $\vec{U}$ is known only implicitly as a solution of the Navier-Stokes system (3.88), we will show that scaling arguments allow us to identify which components of $\mathcal{P}$ are dominating. This will give a link between the tangential and normal aspects of the flow. We first observe that

$$
A^{-t} \partial_{\vec{X}} \vec{U} A^{-1}=A^{-t} \partial_{\vec{\xi}} \vec{U}=\partial_{\vec{\xi}}\left(A^{-t} \vec{U}\right)-\left(\partial_{\vec{\xi}} A^{-t}\right) \vec{U}
$$

By (3.94) we then have

$$
\left(A^{-t} \partial_{\vec{X}} \vec{U} A^{-1}\right)_{i j}=\nu \partial_{\vec{\xi}_{j}} \vec{W}_{i}-\nu\left(\partial_{\vec{\xi}_{i} \vec{\xi}_{j}}^{2} \vec{X}^{t}\right) A^{t} \vec{W}
$$

for all $i, j=1, \ldots, N+1$. Note that we already computed $A\left(\partial_{\vec{\xi}} A^{-1}\right)$ in (3.26). This formula gives for the components of $\mathcal{P}$, see (3.96),

$$
\left\{\begin{aligned}
\mathbf{Z} & =\nu \partial_{\bar{\xi}} \mathbf{W}+\nu \nabla_{\boldsymbol{\xi}} \bar{W}-2 \nu\left(\nabla_{\boldsymbol{\xi}} \mathbf{s}^{t}\right)\left(\partial_{\boldsymbol{\xi}} \mathbf{X}\right)^{-t} \mathbf{W}, \\
f & =2 \nu \partial_{\bar{\xi}} \bar{W} .
\end{aligned}\right.
$$

We do not provide the details of $\mathbf{P}$ since they will not be needed. Note, however, that $\mathbf{P}$ contains only derivatives of $\mathbf{W}$ in $\boldsymbol{\xi}$, not in $\bar{\xi}$.

In the asymptotics $\nu \rightarrow 0$, the boundary conditions (3.92) and (3.93) can only induce boundary layers in the variable $\bar{\xi}$, and as usual in parabolic problems, their length is of order $\sqrt{\nu t}$. Since by assumption $\nu=o\left(\epsilon^{2}\right)$, this length is much smaller than $h$, and we have the situation drawn in Figure 3.2. Thus derivatives in $\boldsymbol{\xi}$ never give singularities, while derivatives in $\bar{\xi}$ and $t$ can be unbounded. However, according to the parabolic scaling we have that $\nu \partial_{\bar{\xi}} \vec{U}$ and $\nu \partial_{t} \vec{U}$, and hence $\nu \partial_{\bar{\xi}} \vec{W}$ and $\nu \partial_{t} \vec{W}$, are bounded. Recall that the geometry is assumed to be sufficiently smooth. Then we can use the incompressibility constraint (3.20) and (3.35) to conclude that $\partial_{\bar{\xi}} \bar{W}$ must be bounded as well. These heuristics and (3.96), (3.103) imply that $\mathcal{P}$ simplifies to

$$
\mathcal{P}=\left(\begin{array}{cc}
0 & \nu \partial_{\bar{\xi}} \mathbf{W} \\
\nu \partial_{\bar{\xi}} \mathbf{W}^{t} & 0
\end{array}\right)+\mathcal{O}(\nu)
$$

Consider now (3.98). By the asymptotics just given, we can write

$$
\nabla_{\boldsymbol{\xi}} \cdot(J M \mathbf{Z})=\nabla_{\boldsymbol{\xi}} \cdot\left(J M\left(\nu \partial_{\bar{\xi}} \mathbf{W}\right)\right)+\mathcal{O}(\nu) .
$$




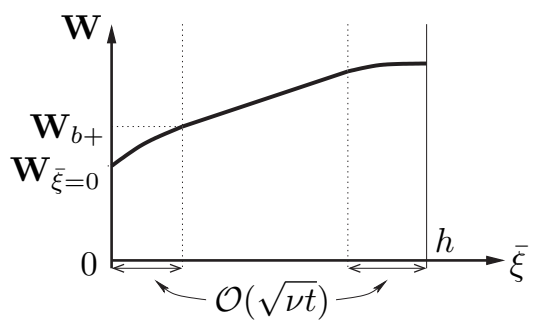

FIG. 3.2. Viscous and material layers

We use again the incompressibility (3.20) and $\mathbf{V}=M \mathbf{W}$ to compute

$$
\begin{aligned}
& \nabla_{\boldsymbol{\xi}} \cdot\left(J M\left(\nu \partial_{\bar{\xi}} \mathbf{W}\right)\right) \\
& =\nu \partial_{\bar{\xi}}\left(\nabla_{\boldsymbol{\xi}} \cdot(J \mathbf{V})\right)-\nu \nabla_{\boldsymbol{\xi}} \cdot\left(\partial_{\bar{\xi}}(J M) \mathbf{W}\right) \\
& =-\partial_{\bar{\xi}}\left(J\left(\nu \partial_{\bar{\xi}} \bar{W}\right)\right)-\nu \partial_{\bar{\xi}}\left(\left(\partial_{\bar{\xi}} J\right) \bar{W}\right)-\nu \nabla_{\boldsymbol{\xi}} \cdot\left(\partial_{\bar{\xi}}(J M) \mathbf{W}\right) .
\end{aligned}
$$

The last two terms on the right-hand side are in $\mathcal{O}(\nu)$. We can therefore conclude that the error terms, which (3.98) induces in (3.74), are all of the form $\partial_{\bar{\xi}} \mathcal{O}(\nu)+\mathcal{O}(\nu)$. Since $\nu=o\left(\epsilon^{2}\right)$ and since $P=0$ for $\bar{\xi}=h(t, \boldsymbol{\xi})$ by condition (3.93), we deduce that the approximation (3.75) is still valid: for $0<\bar{\xi}<h(t, \boldsymbol{\xi})$ we have

$$
P+g(\bar{\xi} c+z)=\frac{1}{2} \mathbf{W}^{t}\left(M_{h}-M\right) \mathbf{W}+g(h c+z)+\mathcal{O}\left(\epsilon^{2}\right) .
$$

In (3.100) the two integrals with $\mathbf{P}$ are of order $o\left(\epsilon^{3}\right)$ and can therefore be neglected. The same is true for $\mathbf{P}_{h} \nabla_{\boldsymbol{\xi}} h$ since $\nabla_{\boldsymbol{\xi}} h$ is in $\mathcal{O}(\epsilon)$. Using now identity (3.107) and the expansion of $\mathbf{W}$ outside the boundary layer, see (3.73), we obtain the conservative momentum equation (2.41) with the extra term $J_{h} \mathbf{Z}_{h}-J_{0} \mathbf{Z}_{0}$ on the right-hand side, up to errors in $\mathcal{O}\left(\epsilon^{3}\right)$ (the boundary layers are of size $\sqrt{\nu t}$, hence negligible in the integrals). We refer to Subsection 3.4 for more details.

Let us now consider the boundary conditions which must finally give the friction. We first note that $A \vec{n}=A^{-t} \vec{n}=\left(\begin{array}{l}0 \\ 1\end{array}\right)$, thus $\vec{U} \cdot \vec{n}=\bar{W}$. Then we multiply the second equation in (3.92) by $A^{-t}$. Using $\sigma=A^{t} \mathcal{P} A$ and the decomposition of $\mathcal{P}$ in (3.96) we find

$$
A^{-t} \sigma \vec{n}=\left(\begin{array}{c}
\mathbf{Z} \\
f
\end{array}\right) \text { and } \vec{n} \cdot \sigma \vec{n}=f
$$

Definition (3.91) gives

$$
A^{-t}\left(\sigma_{T} \vec{n}-\left(\vec{n} \cdot \sigma_{T} \vec{n}\right) \vec{n}\right)=-\left(\begin{array}{c}
\mathbf{Z} \\
0
\end{array}\right)
$$

for the left-hand side of (3.92). Now we observe that the normal component of the right-hand side vanishes because of (3.94) and $\bar{W}=0$ for $\bar{\xi}=0$. Also $|\vec{U}|^{2}=\mathbf{W}^{t} M \mathbf{W}$, see (3.36). Thus with (3.104)

$$
\nu \partial_{\bar{\xi}} \mathbf{W}=\frac{\mu \mathbf{W}}{\sqrt{\mathbf{W}^{t} M \mathbf{W}}} P_{+}+\mathcal{O}(\nu) \quad \text { for } \bar{\xi}=0,
$$


which is $\mathcal{O}(\nu)$ since by assumption $\mu=\mathcal{O}(\nu / \epsilon)$ and $P=\mathcal{O}(\epsilon)$.

To compute the unit normal vector $\vec{N}$ at the free boundary we first note that the tangent space is spanned by the vectors

$$
\left.\vec{T}_{i} \equiv\left(\begin{array}{c}
\mathrm{Id} \\
\frac{1}{c} \mathbf{s}^{t}
\end{array}\right) \partial_{\boldsymbol{\xi}_{i}} \mathbf{X}\right|_{\bar{\xi}=h}+\vec{n} \partial_{\boldsymbol{\xi}_{i}} h \quad \text { for } i=1, \ldots, N .
$$

Indeed, since the two summands are orthogonal to each other, and since $h$ is assumed small enough such that $\partial_{\xi} \mathbf{X}$ has full rank, the $\vec{T}_{i}$ are linear independent. Therefore the normal vector $\vec{N}$ is uniquely defined, up to orientation, by the condition $\vec{N} \perp \vec{T}_{i}$ for all $i$. We put

$$
\eta \vec{N} \equiv-\left.\left(\begin{array}{c}
\mathrm{Id}-\mathbf{s s}^{t} \\
c \mathbf{s}^{t}
\end{array}\right)\left(\partial_{\boldsymbol{\xi}} \mathbf{X}\right)^{-t}\right|_{\vec{\xi}=h} \nabla_{\boldsymbol{\xi}} h+\vec{n}
$$

with normalizing factor $\eta$ given by

$$
\eta^{2} \equiv 1+\left(\nabla_{\boldsymbol{\xi}} h\right)^{t} M_{h} \nabla_{\boldsymbol{\xi}} h .
$$

Note that the matrix $M_{h}$ is positive definite, see (3.6), so $\eta$ is always different from zero. We now compute

$$
\eta A \vec{N}=\left(\begin{array}{c}
-M_{h} \nabla_{\boldsymbol{\xi}} h \\
1
\end{array}\right) \quad \text { and } \quad \eta A^{-t} \vec{N}=\left(\begin{array}{c}
-\nabla_{\boldsymbol{\xi}} h \\
1
\end{array}\right) .
$$

Then $\sigma=A^{t} \mathcal{P} A$ and (3.104) implies

$$
\left.\begin{array}{c}
\eta A^{-t} \sigma \vec{N}=\left(\begin{array}{c}
\nu \partial_{\bar{\xi}} \mathbf{W} \\
-\nu \partial_{\bar{\xi}} \mathbf{W}^{t} M \nabla_{\boldsymbol{\xi}} h
\end{array}\right)+\mathcal{O}(\nu) \\
\eta^{2} \vec{N} \cdot \sigma \vec{N}=-2 \nu \partial_{\bar{\xi}} \mathbf{W}^{t} M \nabla_{\boldsymbol{\xi}} h+\mathcal{O}(\nu)
\end{array}\right\} \quad \text { for } \bar{\xi}=h(t, \boldsymbol{\xi})
$$

Multiplying the second equation in (3.93) by $A^{-t}$ yields, with (3.91),

$$
\begin{aligned}
& \left(1+\left(\nabla_{\boldsymbol{\xi}} h\right)^{t} M \nabla_{\boldsymbol{\xi}} h\right) \nu \partial_{\bar{\xi}} \mathbf{W}-2\left(\nu \partial_{\bar{\xi}} \mathbf{W}^{t} M \nabla_{\boldsymbol{\xi}} h\right) \nabla_{\boldsymbol{\xi}} h=\mathcal{O}(\nu) \\
& \left.\left(\nu \partial_{\bar{\xi}} \mathbf{W}^{t} M \nabla_{\boldsymbol{\xi}} h\right)\left(1-\left(\nabla_{\boldsymbol{\xi}} h\right)^{t} M \nabla_{\boldsymbol{\xi}} h\right)=\mathcal{O}(\nu)\right\} \\
& \text { for } \bar{\xi}=h(t, \boldsymbol{\xi}) \text {. }
\end{aligned}
$$

Since $\nabla_{\boldsymbol{\xi}} h=\mathcal{O}(\epsilon)$ the second equation gives $\nu \partial_{\bar{\xi}} \mathbf{W}^{t} M \nabla_{\boldsymbol{\xi}} h=\mathcal{O}(\nu)$, and using this estimate in the first equation implies $\nu \partial_{\bar{\xi}} \mathbf{W}=\mathcal{O}(\nu)$. From this and the estimate for (3.110) we obtain the improved bound

$$
\partial_{\bar{\xi}} \mathbf{W} \text { is bounded, }
$$

which allows to make precise the expansions we have done and in particular the terms in $\mathcal{O}(\nu)$. We shall denote by $\mathcal{O}_{r}(\nu)$ any function that can be written as $\nu$ times a function which is bounded and has a bounded derivative in $\bar{\xi}$. We observe from (3.102) that the error in $(3.104)$ is $\mathcal{O}_{r}(\nu)$ instead of $\mathcal{O}(\nu)$. Then, the same improvement occurs in (3.97), (3.98) and (3.99). We deduce that the error in (3.100) is of order $\mathcal{O}(\nu \epsilon)$.

Now we analyze more precisely the boundary layer. In (3.97), the leading operator as $\nu \rightarrow 0$ is $\partial_{t} \mathbf{W}-\partial_{\bar{\xi}}\left(\nu \partial_{\bar{\xi}} \mathbf{W}\right)$, thus one expects an asymptotic behavior of the form

$$
\mathbf{W} \sim \varphi\left(t, \frac{t}{\nu}, \boldsymbol{\xi}, \frac{\bar{\xi}}{\nu}\right) \quad \text { in the boundary layer as } \nu \rightarrow 0,
$$


for some smooth function $\varphi$. Looking at the behavior of the boundary layer profile (3.118) solving the heat equation $\partial_{t} \mathbf{W}-\partial_{\bar{\xi}}\left(\nu \partial_{\bar{\xi}} \mathbf{W}\right)=0$ with boundary condition (3.110) (the error in $\mathcal{O}(\nu)$ comes from a term in $\mathcal{O}_{r}(\nu)$ and therefore does not come into play), we deduce the behavior of $\mathbf{W}$ at $\bar{\xi}=0$ as $\nu \rightarrow 0$,

$$
\begin{gathered}
\left.\mathbf{W}\right|_{\bar{\xi}=0} \sim \mathbf{W}_{b+}\left(1-\frac{\sqrt{\nu t}}{\nu} \frac{\left.\mu P_{+}\right|_{\bar{\xi}=0}}{\sqrt{\mathbf{W}_{b+}^{t} M_{0} \mathbf{W}_{b+}}}\right)_{+}, \\
\left.\nu \partial_{\bar{\xi}} \mathbf{W}\right|_{\bar{\xi}=0} \sim \mathbf{W}_{b+} \min \left(\frac{\nu}{\sqrt{\nu t}}, \frac{\left.\mu P_{+}\right|_{\bar{\xi}=0}}{\sqrt{\mathbf{W}_{b+}^{t} M_{0} \mathbf{W}_{b+}}}\right),
\end{gathered}
$$

with $\mathbf{W}_{b+}$ the value at the right of the boundary layer, see Figure 3.2. Now, since by assumption $\mu=\mathcal{O}(\nu / \epsilon)$ and $P=\mathcal{O}(\epsilon)$, the min in (3.120) is realized by the second argument. But from (3.107),

$$
\left.P\right|_{\bar{\xi}=0}=g h c+\left.\left.\frac{1}{2} \mathbf{W}^{t}\right|_{\bar{\xi}=0}\left(M_{h}-M_{0}\right) \mathbf{W}\right|_{\bar{\xi}=0}+\mathcal{O}\left(\epsilon^{2}\right)
$$

and we could replace $\left.\mathbf{W}\right|_{\bar{\xi}=0}$ by $\mathbf{W}_{b+}$ or any value of $\mathbf{W}$ since their difference is at most of order $\mathcal{O}(\epsilon)$. Similarly we can replace $\mathbf{W}_{b+}$ by any value of $\mathbf{W}$ in (3.120), this gives an error in $\mathcal{O}(\nu \epsilon)$ only. This yields the friction term in (2.59). The only bad term is the boundary term at $\bar{\xi}=h$ in (3.100) since $\partial_{\bar{\xi}} \mathbf{W}=W^{1}+\mathcal{O}(\epsilon)$. This is why we have the assumption that $\operatorname{curl} W=\mathcal{O}(\epsilon)$ in Theorem 2.5, that enables to take coherently $W^{1}=0$. Concerning Theorem 2.4, it is possible to consider solutions such that $W^{1}=0$ without any further assumption, see Subsection 3.3.2. The proof is complete.

Proof of Lemma 3.1. For any vector field $\vec{Z}$ we have

$$
J \nabla_{\vec{X}} \cdot \vec{Z}=\nabla_{\vec{\xi}} \cdot(J A \vec{Z}),
$$

by the divergence chain rule. Applying (3.122) to each row of $\sigma$ gives

$$
J \sum_{j} \partial_{\vec{X}_{j}} \sigma_{i j}=\sum_{j} \partial_{\vec{\xi}_{j}}\left(J \sum_{k} A_{j k} \sigma_{i k}\right),
$$

for all indices $i=1, \ldots, N$. Then,

$$
\begin{aligned}
& J \sum_{i} A_{l i}^{-t} \sum_{j} \partial_{\vec{X}_{j}} \sigma_{i j} \\
& =\sum_{i j} A_{l i}^{-t} \partial_{\vec{\xi}_{j}}\left(J \sum_{k} \sigma_{i k} A_{k j}^{t}\right) \\
& =\sum_{i j} \partial_{\vec{\xi}_{j}}\left(J A_{l i}^{-t} \sum_{k} \sigma_{i k} A_{k j}^{t}\right)-J \sum_{i j k}\left(\partial_{\vec{\xi}_{j}} A_{l i}^{-t}\right) \sigma_{i k} A_{k j}^{t}
\end{aligned}
$$

Since $\mathcal{P} A A^{t}=A^{-t} \sigma A^{t}$, the first term on the right-hand side corresponds to the first term in (3.95). For the last term, we can write

$$
\sum_{j}\left(\partial_{\vec{\xi}_{j}} A_{l i}^{-t}\right) A_{k j}^{t}=\sum_{j}\left(\partial_{\vec{\xi}_{l}} A_{j i}^{-t}\right) A_{k j}^{t}=-\sum_{j} A_{j i}^{-t}\left(\partial_{\vec{\xi}_{l}} A_{k j}^{t}\right)
$$


using the definition of $A^{-1}$, see (3.94). By symmetry of $\mathcal{P}$ we have

$$
\begin{aligned}
& -\sum_{i j k}\left(\partial_{\vec{\xi}_{j}} A_{l i}^{-t}\right) \sigma_{i k} A_{k j}^{t} \\
& =\sum_{i j k} \mathcal{P}_{j i} A_{i k} \partial_{\vec{\xi}_{l}} A_{k j}^{t} \\
& =\frac{1}{2} \sum_{i j k} \mathcal{P}_{j i}\left(A_{i k} \partial_{\vec{\xi}_{l}} A_{k j}^{t}+\left(\partial_{\overrightarrow{\xi_{l}}} A_{i k}\right) A_{k j}^{t}\right)=\frac{1}{2} \mathcal{P}: \partial_{\vec{\xi}_{l}}\left(A A^{t}\right)
\end{aligned}
$$

for indices $l=1, \ldots, N+1$. This gives the result.

\section{Invariance under rotation}

One important feature of our models, which distinguishes them from others discussed in the literature, is their invariance under rotation. In this section we will discuss this property for the system in (2.48)-(2.49) only, but similar arguments apply to all other models. Assume that the topography, given by the height field $z$, is rotated in $\mathbb{R}^{N}$. More precisely, assume there exists a new function $\tilde{z}(x)$ with

$$
\tilde{z}(x)=z(R x) \quad \text { for some rotation } R .
$$

For any function $\varphi$, the chain rule gives

$$
\nabla_{x} \tilde{\varphi}(x)=\left.R^{t} \nabla_{y} \varphi(y)\right|_{y=R x},
$$

where $\tilde{\varphi}(x) \equiv \varphi(R x)$ with $x \in \mathbb{R}^{N}$. From this formula we obtain

$$
\tilde{\mathbf{s}}(x)=R^{t} \mathbf{s}(R x) \quad \text { and } \quad \tilde{c}(x)=c(R x) .
$$

The curvature transforms as

$$
\tilde{\mathcal{H}}(x)=R^{t} \mathcal{H}(R x) R .
$$

We will show that a suitable rotation of any solution of (2.48)-(2.49) is a solution of the rotated problem, i.e., of the same equations with the geometrical functions $z$, $\mathbf{s}, c$ and $\mathcal{H}$ replaced by the corresponding tilded ones. So let $(h, \mathcal{V})$ be a solution of (2.48)-(2.49) and consider

$$
\left\{\begin{array}{l}
\tilde{h}(t, x) \equiv h(t, R x) \\
\tilde{\mathcal{V}}(t, x) \equiv R^{t} \mathcal{V}(t, R x) .
\end{array}\right.
$$

Then (4.2) and orthogonality of $R$ yield

$$
\begin{aligned}
\left.\nabla_{x} \cdot(\tilde{h} \tilde{\mathcal{V}} / \tilde{c})\right|_{(t, x)} & =\left.R^{t} \nabla_{y} \cdot R^{t}(h \mathcal{V} / c)\right|_{(t, y=R x)} \\
& =\left.\nabla_{y} \cdot(h \mathcal{V} / c)\right|_{(t, y=R x)} .
\end{aligned}
$$

Thus we find the modified continuity equation

$$
\partial_{t}(\tilde{h} / \tilde{c})+\left.\nabla_{x} \cdot(\tilde{h} \tilde{\mathcal{V}} / \tilde{c})\right|_{(t, x)}=\partial_{t}(h / c)+\left.\nabla_{y} \cdot(h \mathcal{V} / c)\right|_{(t, y=R x)}=0
$$


see (2.48). For the momentum equation we proceed in a similar way. The material derivative, for example, transforms as

$$
\begin{aligned}
\left.\left(\tilde{\mathcal{V}} \cdot \nabla_{x}\right) \tilde{\mathcal{V}}\right|_{(t, x)} & =\left.\left(R^{t} \mathcal{V} \cdot R^{t} \nabla_{y}\right) R^{t} \mathcal{V}\right|_{(t, y=R x)} \\
& =\left.R^{t}\left\{\left(\mathcal{V} \cdot \nabla_{y}\right) \mathcal{V}\right\}\right|_{(t, y=R x)}
\end{aligned}
$$

Using (4.3) and the orthogonality of $R$ we obtain

$$
\begin{aligned}
& \partial_{t} \tilde{\mathcal{V}}+\left(\tilde{\mathcal{V}} \cdot \nabla_{x}\right) \tilde{\mathcal{V}}+\left.\left(\operatorname{Id}-\tilde{\mathbf{s}} \tilde{\mathbf{s}}^{t}\right) \nabla_{x}(g(\tilde{h} \tilde{c}+\tilde{z}))\right|_{(t, x)} \\
& =\left.R^{t}\left\{\partial_{t} \mathcal{V}+\left(\mathcal{V} \cdot \nabla_{y}\right) \mathcal{V}+\left(\operatorname{Id}-\mathbf{s s}^{t}\right) \nabla_{y}(g(h c+z))\right\}\right|_{(t, y=R x)}
\end{aligned}
$$

for the left-hand side of (2.49) and

$$
\begin{aligned}
& -\frac{1}{\tilde{c}^{2}}\left(\tilde{\mathcal{V}}^{t} \tilde{\mathcal{H}} \tilde{\mathcal{V}}\right) \tilde{\mathbf{s}}-\left.\frac{g \mu \tilde{c} \tilde{\mathcal{V}}}{\sqrt{|\tilde{\mathcal{V}}|^{2}+\left(\tilde{\mathbf{s}}^{t} \tilde{\mathcal{V}} / \tilde{c}\right)^{2}}}\left(1+\frac{\tilde{\mathcal{V}}^{t} \tilde{\mathcal{H}} \tilde{\mathcal{V}}}{g \tilde{c}^{3}}\right)\right|_{(t, x)} \\
& =\left.R^{t}\left\{-\frac{1}{c^{2}}\left(\mathcal{V}^{t} \mathcal{H} \mathcal{V}\right) \mathbf{s}-\frac{g \mu c \mathcal{V}}{\sqrt{|\mathcal{V}|^{2}+\left(\mathbf{s}^{t} \mathcal{V} / c\right)^{2}}}\left(1+\frac{\mathcal{V}^{t} \mathcal{H} \mathcal{V}}{g c^{3}}\right)\right\}\right|_{(t, y=R x)}
\end{aligned}
$$

for the right-hand side. This proves the rotational invariance of (2.49).

\section{REFERENCES}

[1] D. Bresch and B. Desjardins, On a viscous shallow water equation (Saint Venant model) and its quasi-geostrophic limit. Preprint 2002.

[2] F. Bouchut, A. Mangeney-Castelnau, B. Perthame, and J.-P. Vilotte, A new model of Saint Venant and Savage-Hutter type for gravity driven shallow water flows. C.R. Acad. Sci. Paris, série I 336, 531-536, 2003.

[3] J.-F. Gerbeau and B. Perthame, Derivation of viscous Saint-Venant system for laminar shallow water; Numerical validation, Discrete and Continuous Dynamical Systems, Series B, 1, 89-102, 2001.

[4] J. M. N. T. Gray, Y.-C. Tai, and S. Noelle, Shock waves, dead zones and particle-free regions in rapid granular free-surface flows. J. Fluid Mech. 491, 161-181, 2003.

[5] J. M. N. T. Gray, M. Wieland, and K. Hutter, Gravity driven free surface flows for granular avalanches over complex basal topographies. Proc. R. Soc. Lond. A 455, 1841-1874, 1999.

[6] P. Gwiazda, An existence result for a model of granular material with non-constant density. Asympt. Anal. 30, 43-60, 2002.

[7] P.-L. Lions, Mathematical Topics in Fluid Mechanics, Incompressible Models. Oxford Lecture Series in Mathematics and its Applications, vol. 1, Oxford University Press, 1996.

[8] P.-L. Lions, B. Perthame, and P. E. Souganidis, Existence and stability of entropy solutions for the hyperbolic systems of isentropic gas dynamics in Eulerian and Lagrangian coordinates. Comm. Pure Appl. Math. 49, 599-638, 1996.

[9] O. Pouliquen and Y. Forterre, Friction law for dense granular flows: application to the motion of a mass down a rough inclined plane. J. Fluid Mech. 453, 133-151, 2002.

[10] S. P. Pusadaini, Dynamics of flow avalanches over curved and twisted channels: theory, numerics and experimental validation. Dissertation, Fachbereich Mechanik, Technische Universität Darmstadt. Available at: http://elib.tu-darmstadt.de/diss/000393.

[11] S. P. Pusadaini and K. Hutter, Granular avalanche model in arbitrarily curved and twisted mountain terrain: a basis for the extension to debris flow. In: Debris-Flow Hazards Mitigation: Mechanics, Predictions and Assessment (Eds. D. Rickenmann and C.-L. Chen). Volume 1, 491-502, Millpress. 
[12] A. J. C. de Saint-Venant, Théorie du mouvement non-permanent des eaux, avec application aux crues des rivières et à l'introduction des marées dans leur lits. C. R. Acad. Sc. Paris 73, $147-154,1871$.

[13] S. B. Savage and K. Hutter, The dynamics of avalanches of granular materials from initiation to run-out. Acta Mech. 86, 201-223, 1991.

[14] M. Wieland, J. M. N. T. Gray, and K. Hutter, Channelized free-surface flow of cohesionless granular avalanches in a chute with shallow lateral curvature. J. Fluid Mech. 392, 73-100, 1999. 